\title{
Review Article \\ Function, Role, and Clinical Application of MicroRNAs in Vascular Aging
}

\author{
Xiao Lin, Jun-Kun Zhan, Yan-Jiao Wang, Pan Tan, Yi-Yin Chen, \\ Hui-Qian Deng, and You-Shuo Liu \\ Department of Geriatrics, The Second Xiangya Hospital, Central South University, Changsha, Hunan 410011, China \\ Correspondence should be addressed to You-Shuo Liu; liuyoushuo@yeah.net
}

Received 10 August 2016; Revised 7 November 2016; Accepted 23 November 2016

Academic Editor: Vinicio A. de Jesus Perez

Copyright (c) 2016 Xiao Lin et al. This is an open access article distributed under the Creative Commons Attribution License, which permits unrestricted use, distribution, and reproduction in any medium, provided the original work is properly cited.

\begin{abstract}
Vascular aging, a specific type of organic aging, is related to age-dependent changes in the vasculature, including atherosclerotic plaques, arterial stiffness, fibrosis, and increased intimal thickening. Vascular aging could influence the threshold, process, and severity of various cardiovascular diseases, thus making it one of the most important risk factors in the high mortality of cardiovascular diseases. As endothelial cells (ECs) and vascular smooth muscle cells (VSMCs) are the main cell biological basis of these pathology changes of the vasculature, the structure and function of ECs and VSMCs play a key role in vascular aging. MicroRNAs (miRNAs), small noncoding RNAs, have been shown to regulate the expression of multiple messenger RNAs (mRNAs) posttranscriptionally, contributing to many crucial aspects of cell biology. Recently, miRNAs with functions associated with aging or aging-related diseases have been studied. In this review, we will summarize the reported role of miRNAs in the process of vascular aging with special emphasis on EC and VSMC functions. In addition, the potential application of miRNAs to clinical practice for the diagnosis and treatment of cardiovascular diseases will also be discussed.
\end{abstract}

\section{Introduction}

Aging is a multifactorial process characterized by a progressive loss of physiological integrity and functionality, which increases mortality and susceptibility to diseases, including cardiovascular diseases, diabetes, osteoporosis, immunological diseases, various neurodegenerative diseases, and cancer [1-3]. Vascular aging is a specific type of organic aging playing a key role in the process of overall aging. Vascular aging is one of the most important risk factors in the high mortality of cardiovascular diseases and could influence the threshold, process, and severity of various cardiovascular diseases. Vascular aging is tightly linked to alterations in the biological functions and structural properties of the vascular wall, mainly including endothelial cells (ECs) and vascular smooth muscle cells (VSMCs). ECs, the inner layer of blood vessels, on the one hand, serve as a barrier between the blood stream and vessel and, on the other hand, regulate many aspects of vessel function, such as the control of vasodilation and vasoconstriction, inhibition of the adhesion of leukocytes and platelets to prevent blood coagulation, and suppression of vessel wall hypertrophy by inhibiting VSMC proliferation [4]. Furthermore, structural damage and dysfunction of ECs, such as senescence, apoptosis, proliferation, and inflammation, are closely associated with vascular aging. VSMCs, the main cells of the media vessel wall, can control blood flow by contracting or relaxing in response to external stimuli and play an important role in vascular pathologies. With increasing age, VSMCs are thought to undergo a phenotypic change from the quiescent, contractile phenotype to a synthetic phenotype. This synthetic phenotype is capable of migration into the intima and subsequent proliferation and extracellular matrix (ECM) synthesis, which in turn affects vascular function and disease outcome in the elderly [5]. Because vessels serve as transportation tools, they could supply nutrients, oxygen, and active substances and remove wastes or byproducts and carbon dioxide produced in tissues [6]. Thus, vessels are critical to maintaining physiological homeostasis in vivo; as William Osler said, "a man is just as old as his arteries" [7]. Therefore, a better understanding of vascular 
physiological and functional changes with aging is necessary and crucial to combat cardiovascular diseases in the future.

MicroRNAs (miRNAs), which are small noncoding RNAs, are approximately 18-25 nucleotides long. miRNA genes are mainly transcribed by RNA polymerase. They are initially transcribed as large precursors, called primary miRNAs (pri-miRNAs). Pri-miRNAs are then processed by the RNase-III enzymes Drosha and Dicer to generate mature miRNA products. Recently, studies have shown that miRNAs could cause target mRNA degradation at the posttranscriptional level and/or suppress the translation of mRNA into protein via interaction with the $3^{\prime}$-untranslated region ( $3^{\prime}$ UTR) of target mRNA by complementary base pairing [8]. So far, over 1000 miRNAs have been found in human cells. Each miRNA might target several genes and different miRNAs might target the same gene. As negative regulators of gene regulation, miRNAs contribute to many essential physiological and pathophysiological processes in humans, including differentiation, proliferation, apoptosis, migration, homeostasis, and various diseases $[9,10]$. Therefore, it is not surprising that miRNAs are also involved in vascular aging and age-related diseases because of their multiple biological functions $[11,12]$.

\section{2. miRNAs and Vascular Aging}

Increasing evidence has shown that aging-associated physiological and functional disorders are associated with alterations in miRNAs, suggesting that miRNAs are novel cellular senescence regulators $[59,60]$. Vascular aging is tightly linked to alterations in the biomechanical and structural properties of the vascular wall, including ECs and VSMCs dysfunction or apoptosis as well as increased arterial stiffness [11]. Until now, however, only the functions of a few miRNAs have been associated with cell dysfunction and/or vascular aging. In this section, we will discuss the role of miRNAs in the progression of vascular aging.

2.1. miRNAs and Endothelial Function. The vascular endothelium, a thin layer of ECs that lines the inner surface of blood vessels, is a critical interface between blood and all tissues. When the endothelium is exposed to various stimuli, such as hypoxia, proinflammatory cytokines, oxidative stress, hypertension, hyperglycemia, shear stress, aging, or injury, the function of ECs will be influenced, which is related to the proliferation, apoptosis, migration, senescence, angiogenesis, and inflammation of ECs [61]. Here, we will focus on individual miRNAs associated with endothelial functions (Table 1).

2.1.1. Influence of miRNAs on EC Apoptosis. EC apoptosis plays a vital role in the initiation and progression of atherosclerotic. In addition, EC apoptosis is responsible for plaque instability because EC death can predispose to arterial thrombosis, which could cause acute coronary occlusion and sudden death [62]. Accumulating evidence has indicated that miRNAs act as critical regulators to participate in EC apoptosis.

Several miRNAs are involved in the regulatory mechanisms of cellular apoptosis of ECs. Some are antiapoptotic
miRNAs while others have proapoptotic effects. miR-126 was the most abundant miRNA in apoptotic bodies derived from ECs. It induced CXCL12 expression by targeting RGS16 and protected mice from atherosclerosis in a miR-126-dependent manner [63]. Recently, Chen et al. also demonstrated that miR-126 inhibits vascular ECs apoptosis through targeting PI3K/Akt signaling pathway [15]. miR-495 targets CCL2, significantly promoting human umbilical vein endothelial cell (HUVEC) proliferation and inhibiting apoptosis by affecting the expression of cleaved caspase-3 [19]. In addition, miR-19b plays a key role in the attenuation of TNF- $\alpha$ induced EC apoptosis, and this function is closely linked to the Apaf1/caspase-7-dependent pathway [22]. Nevertheless, miR-132 promoted apoptosis of HUVEC induced by TNF$\alpha$ and inhibited its proliferation, viability, and migration by inhibiting SIRT1 [23].

Oxidatively modified low density lipoprotein (Ox-LDL) is a major risk factor in the development of atherosclerosis. miR-365 and miR-US25-1 exerted a proapoptotic function in ox-LDL treated ECs by targeting the inhibition of Bcl-2 and BRCC 3 expression, respectively [26, 27]. Another miRNA, named miR-26a, was sufficient to reverse ox-LDL-induced apoptosis; the underlying mechanisms likely involved repression of TRPC6 and the associated downstream apoptotic pathway [14]. Furthermore, the let-7 family was found to be related to atherosclerosis and coronary artery diseases and is highly expressed in ECs. The inhibitory effects of let-7a and let-7b on ox-LDL induced EC apoptosis and dysfunction are partly obtained through the LOX-1/ROS/p38MAPK/NF- $\kappa \mathrm{B}$ signaling pathway and the LOX-1/ROS/PKB/eNOS pathway [20]. Meanwhile, let-7g negatively regulated apoptosis in ECs by targeting caspase-3 expression [21]. In addition, miR221/222 could partly alleviate apoptotic cell death mediated by ox-LDL through the suppression of Ets-1 and its downstream target, p21 [18].

Both intra- and extracellular conditions, such as shear stress, oxidative stress, hyperglycemia, and withdrawal, have a major effect on miRNA expression in EC functions, and the molecular mechanisms involved have been extensively studied [13, 16, 17, 24]. miR-21 targets PTEN and attenuates endothelial apoptosis by regulating Akt phosphorylation, eNOS phosphorylation, and NO production in human ECs [13]. G $\alpha 12$ protects HUVEC from serum withdrawal-induced apoptosis by retaining miR-155 expression [17]. In diabetes patients, miR-130a inhibits the JNK pathway by targeting MAP3K12, contributing to its antiapoptotic effect and the maintenance of endothelial progenitor cell (EPC) function under high glucose conditions [16]. Other miRNAs have a proapoptotic effect on ECs. For example, miR-200c is upregulated by oxidative stress and induces EC apoptosis and senescence via ZEB1 inhibition [24]. Moreover, platelet-released miR-223 promotes advanced glycation end product- (AGE-) induced vascular EC apoptosis via targeting of IGF-1 [25].

2.1.2. Functions of miRNAs in EC Senescence. Senescence is associated with the cellular response to various environmental stressors and damage, which is defined as permanent cell cycle arrest. Senescent ECs are important in atherosclerosis and other age-related diseases [64]. An EC often undergoes 
TABLE 1: miRNAs implicated in ECs functions.

\begin{tabular}{|c|c|c|c|}
\hline ECs & miRNAs & Targets & Reference \\
\hline \multicolumn{4}{|c|}{ Cellular apoptosis } \\
\hline \multirow{9}{*}{ Inhibit } & miR-21 & PTEN & {$[13]$} \\
\hline & miR-26a & TRPC6 & {$[14]$} \\
\hline & miR-126 & PI3K/Akt & {$[15]$} \\
\hline & miR-130a & MAP3K12 & {$[16]$} \\
\hline & miR-155 & Unknown & [17] \\
\hline & $\operatorname{miR}-221 / 222$ & Ets-1/caspase-7 & {$[18]$} \\
\hline & miR-495 & CCL2 & {$[19]$} \\
\hline & let-7a/b & LOX-1 & {$[20]$} \\
\hline & let-7g & Caspase-3 & {$[21]$} \\
\hline \multirow{6}{*}{ Promote } & miR-19b & Apaf1 & {$[22]$} \\
\hline & miR-132 & SIRT1 & {$[23]$} \\
\hline & miR-200c & ZEB1 & {$[24]$} \\
\hline & miR-223 & IGF-1 & [25] \\
\hline & $\operatorname{miR}-365$ & $\mathrm{Bcl}-2$ & {$[26]$} \\
\hline & miR-US25-1 & BRCC 3 & {$[27]$} \\
\hline \multicolumn{4}{|c|}{ Cellular senescence } \\
\hline \multirow{5}{*}{ Promote } & miR- 22 & Vasohibin-1 & {$[28]$} \\
\hline & miR-34a & SIRT1 & [29] \\
\hline & miR-146a & SIRT1 & {$[30]$} \\
\hline & miR-200c & ZEB1 & {$[24]$} \\
\hline & $\operatorname{miR}-217$ & SIRT1 & {$[31]$} \\
\hline \multirow{2}{*}{ Inhibit } & miR-92a & Unknown & {$[32]$} \\
\hline & let-7g & SIRT1 & {$[33]$} \\
\hline \multicolumn{4}{|c|}{ Cellular proliferation } \\
\hline \multirow{4}{*}{ Promote } & miR-29a & HBP1 & {$[34]$} \\
\hline & miR-126-5p & Dlk1 & [35] \\
\hline & $\mathrm{miR}-487 \mathrm{~b}$ & THBS1 & {$[36]$} \\
\hline & miR-495 & CCL2 & {$[19]$} \\
\hline \multirow{6}{*}{ Inhibit } & miR-21 & RhoB & {$[37]$} \\
\hline & miR-24 & Spl & {$[38]$} \\
\hline & $\operatorname{miR}-34 a$ & SIRT1 & [29] \\
\hline & miR-92a & SIRT1 & [39] \\
\hline & miR-101 & mTOR & {$[40]$} \\
\hline & miR-125a & Bcl-2 & {$[41]$} \\
\hline \multicolumn{4}{|c|}{ Cellular angiogenesis } \\
\hline \multirow{2}{*}{ Proangiogenesis } & miR-92a & PTEN & {$[42]$} \\
\hline & miR-126 & Spred-1 & {$[43]$} \\
\hline \multirow{8}{*}{ Antiangiogenesis } & miR-15a & FGF2 and VEGF & {$[44]$} \\
\hline & miR-20a & MKK3 & {$[45]$} \\
\hline & miR-21 & RhoB & {$[46]$} \\
\hline & miR-351 & STAT3 & {$[47]$} \\
\hline & $\operatorname{miR}-214$ & XBP1 & {$[48]$} \\
\hline & $\operatorname{miR}-223$ & $\beta 1$ integrin & [49] \\
\hline & miR-221/222 & c-Kit & {$[50]$} \\
\hline & miR-106 & STAT3 & {$[51]$} \\
\hline
\end{tabular}


TABLE 1: Continued.

\begin{tabular}{|c|c|c|c|}
\hline ECs & miRNAs & Targets & Reference \\
\hline \multicolumn{4}{|c|}{ Cellular inflammation } \\
\hline \multirow[b]{2}{*}{ Promote } & miR-21 & $\operatorname{PPAR} \alpha$ & {$[52]$} \\
\hline & miR-92a & SOCS5 & {$[53]$} \\
\hline \multirow{6}{*}{ Inhibit } & miR-30-5p & Ang2 & {$[54]$} \\
\hline & miR-126 & VCAM-1 & {$[55]$} \\
\hline & miR-155 & Ang II type 1 receptor & {$[56]$} \\
\hline & miR-181b & NF-kB & [57] \\
\hline & miR-663 & SLC7A5 and NAV2 & {$[58]$} \\
\hline & let-7g & TGF- $\beta$ & [33] \\
\hline
\end{tabular}

EC, endothelial cell; PTEN, phosphatase and TENsin homologue; PI3K: phosphatidylinositol 3-kinase; TRPC6: transient receptor potential canonical 6; MAPK: mitogen-activated protein kinase; Ets-1: E26 transformation-specific 1; CCL2: C-C motif chemokine 2; LOX-1: lectin-like low-density lipoprotein receptor 1; Apaf-1: apoptotic protease-activating factor; SIRT1: silent information regulator 1; ZEB1: zinc finger E-box-binding homeobox 1; IGF-1: insulin-like growth factor-1; BRCC 3: BRCA1-BRCA2-containing complex; HBP1: HMG box-containing protein-1; Dlk1: delta-like 1 homologue; THBS1: thrombospondin 1; Sp1: specificity protein 1; mTOR: mammalian target of rapamycin; FGF2: fibroblast growth factor; VEGF: vascular endothelial growth factor; MKK3: mitogenactivated protein kinase kinases 3; STAT3: signal transducer and activator of transcription 3; XBP-1, a key unfolded protein response transcription factor; PPAR $\alpha$ : peroxisome proliferator-activated receptor- $\alpha$; SOCS5: suppressor of cytokine signaling 5; VCAM-1: vascular cell adhesion molecule 1; NF-kB: nuclear factor-kappa B; TGF- $\beta$ : tumor growth factor- $\beta$.

both replicative and stress-induced presenescence. The function of miRNAs involved in the regulatory mechanisms of ECs senescence has been investigated. During replicative senescence of ECs, miR-22 could accelerate the process of aging by down regulating Vasohibin-1 [28]. However, miR92a, a component of the miR-17-92 cluster, is highly expressed in young ECs. Rippe et al. reported that senescence of human ECs is associated with the reduced expression of miR-92a [32]. In the progress of stress-induced presenescence of ECs, miR-221 promotes senescence of human arterial ECs by inhibiting NO production and activating NF- $\kappa \mathrm{B}$ signaling in human ECs [32]. Increased expression of miR-200c by ROS could induce the cellular senescence target zinc finger E-boxbinding homeobox 1 (ZEB1) [24].

SIRT1 is a longevity gene that protects cells against oxidative and genotoxic stress. Recent studies have indicated that miR-34a is highly expressed in ECs. miR-34a expression is increased in senescent HUVECs and induces HUVEC senescence through the suppression of SIRT1 [29]. Two other miRNAs, miR-217 and miR-146a, promote senescence with a reduction of SIRT1 in ECs [30, 31]. On the contrary, let-7g has the effect of reducing EC senescence by increasing SIRT1 protein levels [33].

2.1.3. miRNAs and EC Proliferation. EC proliferation and viability are critical in the process of promoting endothelial healing and improving vascular function. Numerous lines of evidence support the involvement of miRNAs in EC proliferation. It has been reported that miR-495 significantly promoted HUVEC proliferation by directly targeting CCL2 [19]. Feng et al. demonstrated that miR-487b enhanced cell proliferation and migration in HUVECs through regulating THBS1 [36]. Apart from the influence on EC apoptosis, endothelial miR-126-5p could also promote the proliferation of ECs through suppression of the Notch1 inhibitor deltalike 1 homologue (Dlk1), thereby preventing the formation of atherosclerotic lesions [35]. Another highly expressed
miRNA in endothelium is miR-29a, which was able to accelerate G1 to S cell cycle transition in HUVECs and enforce the expression of miR-29a in endothelium, remarkably promoting cell proliferation and angiogenesis via the targeting of HBP1 [34].

However, some miRNAs also exist that inhibit the proliferation of ECs. Both miR-34a and miR-92a are upregulated in ECs during aging, inhibiting cell proliferation and migration by targeting SIRT1 $[29,39]$. Moreover, miR-21 can enhance the rapamycin-induced inhibition of endothelial proliferation by targeting RhoB [37]. In addition, miR-101 can induce cell cycle arrest at the G1/S transition and suppress mTOR expression and EC proliferation induced by laminar shear stress [40]. HUVEC proliferation is significantly inhibited by miR-125a and miR-24 via regulation of the expression of $\mathrm{Bcl}-2$ and Spl, respectively $[38,41]$.

2.1.4. Effects of miRNAs on Endothelial Angiogenesis. Angiogenesis is the process of new blood vessel and capillary network formation in the body, which is essential for recovery after cardiac and skeletal muscle injury or ischemia. Aged individuals, however, appear to have impaired physiological angiogenesis and are at higher risk of processes associated with pathological vessel formation, whereas ECs play a crucial role in the initiation of angiogenesis and the formation of early vascular structures [65]. A large number of miRNAs are responsible for angiogenesis and are expressed in ECs [43, 49-51, 66]. Wang et al. reported that miR-126, the endothelial specific miRNA, enhances the proangiogenic actions of VEGF and FGF and promotes blood vessel formation by repressing the expression of Spred-1, an intracellular inhibitor of angiogenic signaling [43]. Besides, members of the miRNA-17-92 cluster also exhibit a cellintrinsic antiangiogenic function in ECs [42, 66, 67]. For example, pre-miR-92a treatment improves HUVEC viability and preserves angiogenic capacity under oxidative stress, at least partially through the downregulation of PTEN 
expression [42]. Another study reported that miR-92a was identified as a negative regulator of angiogenesis by targeting the A5 integrin subunit (ITGA5) [67]. The contradictory results between the two studies might be attributed to the different functions of their different target proteins. Furthermore, miR-20a, another component of the miR-17-92 cluster, acts in a feedback loop to repress the expression of MKK3 and to negatively regulate $\mathrm{p} 38$ pathway-mediated VEGF-induced ECs migration and angiogenesis [45].

Other important miRNAs involved in angiogenesis regulation are the so-called antiangiogenic miRNAs, which include miR-221/222, miR-223, miR-206, miR-15a, miR-214, miR-21, miR-106b, miR-129-1, miR-133, miR-29c, miR-217, and miR-351. Poliseno et al. proved that miR-221/222 and miR-223 are antiangiogenic factors and that they affect the expression of the c-Kit receptor and $\beta 1$ integrin in ECs, respectively $[49,50]$. The signal transducer and activator of transcription 3 (STAT3) signaling pathway was regarded as a target for the prevention of atherosclerosis or other cardiovascular diseases. Previous studies showed that both miR-351 and miR-106b were upregulated in atherosclerotic mice and exerted an antiangiogenic effect in ECs by targeting STAT3 in vitro $[47,51]$. Other miRNAs, such as miR-214, miR21, and miR-15a, reduce angiogenesis of HUVEC by directly targeting XBP1, RhoB, and FGF2 and VEGF, respectively $[44,46,48]$. However, some miRNAs influence angiogenesis by affecting other functions of ECs. For example, miR129-1 and miR-133 modulate angiogenesis by suppressing the proliferation rate, cell viability, and migration activity of HUVECs in vitro by targeting VEGFR2 and FGFR1, respectively [68]. Moreover, miR-29c plays a significant role in regulating angiogenic properties of HUVECs through the IGF-1/PI3K/AKT signaling pathway [69].

2.1.5. miRNAs Associated with Endothelial Inflammation. ECs, activated by shear stress, lipopolysaccharides, or cytokines, can modulate the expression of adhesion molecules and chemokines, leukocytes rolling over the endothelium and adhesion to vessels [70], which are stimulators of inflammation. Inflammation is associated with the development and progression of age-related conditions and they make individuals, especially the aged, more susceptible to cardiovascular diseases. Moreover, inflammatory mediators also play a fundamental role in the initiation, progression, and eventual rupture of atherosclerotic plaques and could therefore accelerate vascular aging [71]. Recent reports have shown that miRNAs can control vascular inflammation by controlling leukocyte activation and infiltration through the vascular wall [72]. Loyer et al. reported that miR-92a acts as a proinflammatory regulator in ECs by activating inflammatory cytokines and chemokines and promoting monocyte adhesion [53]. Zhou et al. showed that miR-21 suppresses the translation of peroxisome proliferator-activated receptor $\alpha(\operatorname{PPAR} \alpha)$ mRNA, promoting endothelial inflammation by inducing the expression of vascular cell adhesion protein 1 (VCAM-1) and C-C motif chemokine 2 (CCL2) by increasing the activity of the transcription factor AP-1 [52].

Other important miRNAs associated with inflammation could inhibit endothelial inflammation. Harris et al. found that the inhibition of miR-126 increases proinflammatory TNF- $\alpha$ expression, which activates NF- $\kappa \mathrm{B}$ and interferon regulatory factor 1 and finally induces the expression of VCAM-1 and the adhesion of leukocytes to ECs [55]. The systemic delivery of miR-181b also attenuates atherosclerosis by targeting NF- $\kappa$ B signaling in ECs [57]. miR-663, one of the oscillatory shear-sensitive miRNAs in HUVECs, is involved in oscillatory shear stress-induced cellular inflammation by regulating the potential targets of SLC7A5 and NAV2 [58]. miR-155 inhibits angiotensin II- (Ang II-) induced inflammation, migration, and apoptosis in HUVECs by targeting the Ang II type 1 receptor [56]. miR-30-5p acts in an anti-inflammatory manner in ECs induced by KLF2 and shear stress by impairing the expression of Ang2 and inflammatory cell-cell adhesion molecules [54]. Let-7g decreases EC inflammation and monocyte adhesion and increases angiogenesis via the TGF- $\beta$ pathway [33].

Several important miRNAs regulate different kinds of EC functions among those that participate in the functional regulation of ECs. For example, miRNA-126 can inhibit apoptosis in ECs via the PI3K/AKT signaling pathway [15]. Meanwhile, it also plays a role in promoting angiogenesis and inflammation in ECs [43, 55]. In addition, miR-221/222 is also involved in the regulation of apoptosis, senescence, and angiogenesis in ECs $[18,50]$. Upon summarizing numerous previous studies, it is not difficult to conclude that the SIRT1 gene, initially identified as a longevity gene, plays an important role in the regulation of ECs function. On the one hand, SIRT1 can be regarded as a regulatory target of multiple miRNAs, such as miR-34a, miR-221/222, miR-217, miR-132, and let-7g; on the other hand, it is involved in regulating multiple functions of ECs, such as senescence, apoptosis, and proliferation. Figure 1 shows the network of important miRNAs regulating the function of ECs.

2.2. miRNAs and VSMCs Function. VSMCs, the predominant cells in the tunica media of arteries, are highly specialized cells that represent the main contributor to vessel wall formation and vascular tension maintenance. The predominant phenotype of VSMCs is quiescent/contractile with nonmigratory and nonproliferative in periods of health. However, with the progress of aging and in response to various pathological stimuli, VSMCs deviate from their physiological state and switch to a proliferative, migratory, apoptotic, and differentiation phenotype, which is called phenotypic modulation or switching [73]. Recently, emerging evidence has revealed that miRNAs are involved in vascular disease through the regulation of VSMC migration, proliferation, differentiation, and apoptosis [74-77]. Next, we will summarize the current knowledge on the role of miRNAs in the regulation of VSMCs functions, including proliferation, migration, apoptosis, and differentiation (Figure 2).

2.2.1. miRNAs and VSMCs Apoptosis and Senescence. Apoptosis and senescence of VSMCs have been identified as important processes in a variety of human vascular diseases, such as atherosclerosis [78, 79]. ox-LDL plays an important role in atherogenesis. Studies have shown that hsa-let-7g can inhibit ox-LDL uptake and reduce apoptosis in SMCs 


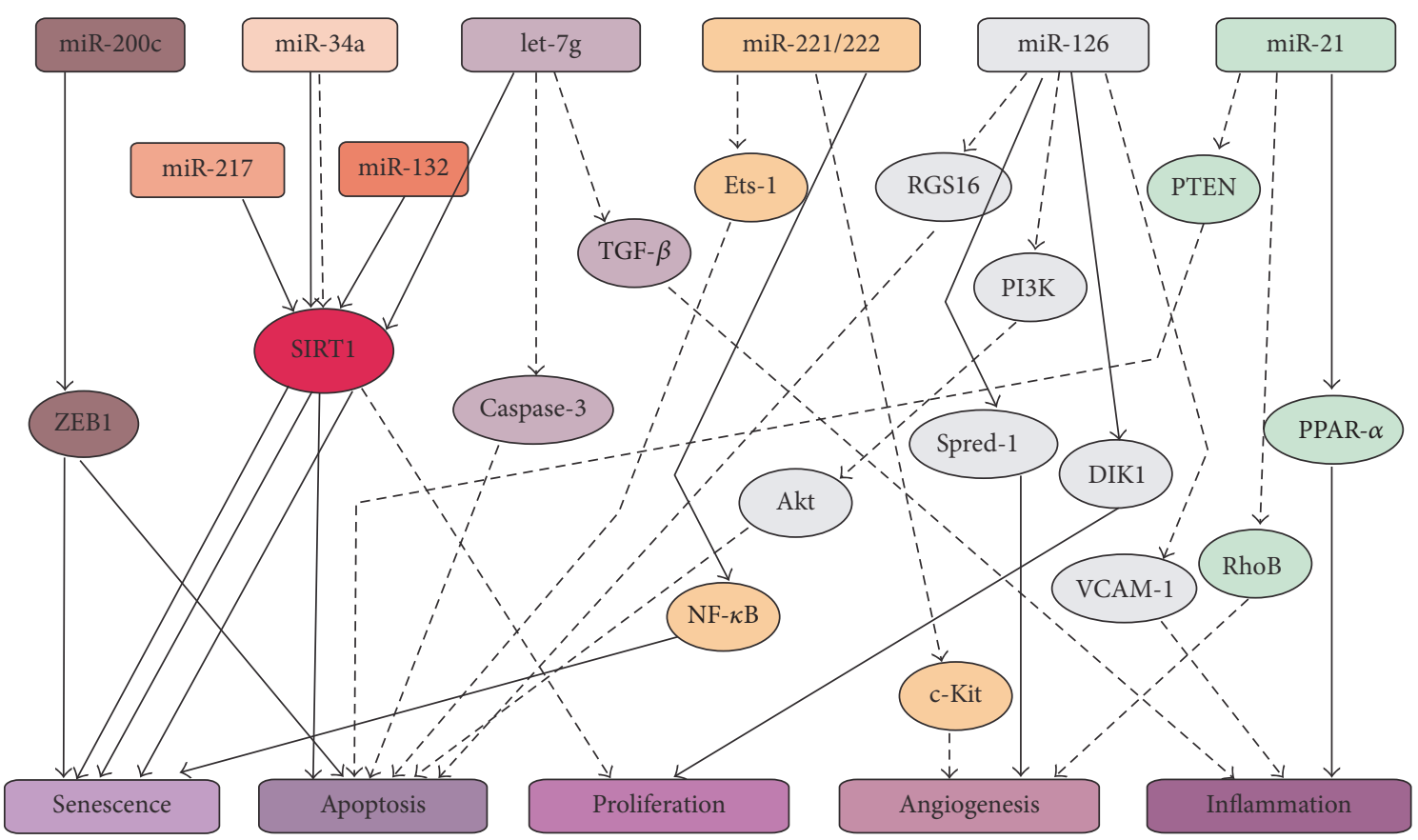

FIGURE 1: Network system of several important miRNAs regulating the function of ECs. The picture shows that SIRT1 is an important gene in the regulation of EC function. miR-34a, miR-217, miR-132, and let-7g are targets of SIRT1. Other miRNAs, such as miR-221/222, miR-126, and miR-21, participate in the function of ECs via targeting different genes. They can promote or inhibit the functions (senescence, apoptosis, proliferation, angiogenesis, and inflammation) of ECs. "-" indicates promotion effects; “--." denotes inhibition effects.

by the downregulation of cytochrome $\mathrm{C}$ and Smac/Diablo and upregulation of Bcl-2 expression [74, 80]. In addition, miR-34a, an aging-associated miRNA, can promote VSMCs senescence and inflammation through SIRT1 downregulation and senescence-associated secretory phenotype factor induction, respectively [76]. Moreover, miR-92a overexpression inhibits $\mathrm{H}_{2} \mathrm{O}_{2}$-induced VSMCs apoptosis and senescence by suppressing both mitogen-activated protein kinase 4 (MKK4) and JNK1 pathways [81]. Another miRNA, miRNA-146a was found to induce VSMC apoptosis via activation of the NF- $\kappa$ B signaling pathway [82].

2.2.2. miRNAs and VSMCs Proliferation and Migration. In the native vessel, VSMCs are maintained in a quiescent/contractile, nonmigratory and nonproliferative state. In response to vascular or mechanical injury, VSMCs switch to the dedifferentiated/synthetic phenotype and increase their ability to migrate to the intima space, proliferate, and produce the ECM, which contributes to the development of atherosclerosis. Therefore, the proliferation and migration of VSMCs are closely associated and together play a central role in the growth of atherosclerotic lesions. An increasing number of studies have demonstrated that miRNAs play an important role in the regulation of VSMC proliferation and migration [83-85].

(1) miRNAs That Promote the Proliferation and Migration of VSMCs. Some miRNAs have been found to promote the proliferation and migration of VSMCs. miR-21 is one of the most abundant miRNAs in the vascular wall following balloon injury; it can enhance VSMCs migration and proliferation caused by TSP-1 [86] and stimulate the proliferation and migration of VSMCs through the suppression of c-Ski [83]. c-Ski is a molecule that is expressed in VSMCs to suppress VSMC stimulation and intimal hyperplasia in a rat balloon injury model [87]. Therefore, in cultured human VSMCs, low expression of miR-21 significantly inhibits cell proliferation and migration by targeting different genes [ 88 , 89]. miR-146a, a novel regulator of VSMC fate, promotes VSMCs proliferation and migration by targeting Krüppellike factor 4 (KLF4) mRNA [90, 91]. Moreover, miR-146a and miR-21 were significantly upregulated in atherosclerotic plaques and cooperated to accelerate VSMC growth and cell cycle progression by targeting Notch2 and Jag1 [92]. Interestingly, miR-221/222, contrary to its effects of antiproliferation, antimigration, and proapoptosis in ECs, had the effects of proproliferation, promigration, and antiapoptosis in VSMCs. The different expression profiles of the target genes p27(Kip1), p57(Kip2), and c-kit between the two cell types might be related to the opposite effects $[85,93]$.

Diabetic VSMCs exhibit significantly increased rates of proliferation and migration, which is the most common pathological change in atherosclerosis. miR-138 promotes the proliferation and migration of VSMCs in $\mathrm{db} / \mathrm{db}$ mice by suppressing the expression of SIRT1 [94], and miR-133a serves as a stimulatory factor for IGF-1R expression by prolonging the half-life of IGF-1R mRNA and promoting IGF-1-induced VSMC proliferation in murine atherosclerosis [95]. Therefore, identification of the miR-138 and miR-133aIGF-1R pathways might provide insight into the design of 


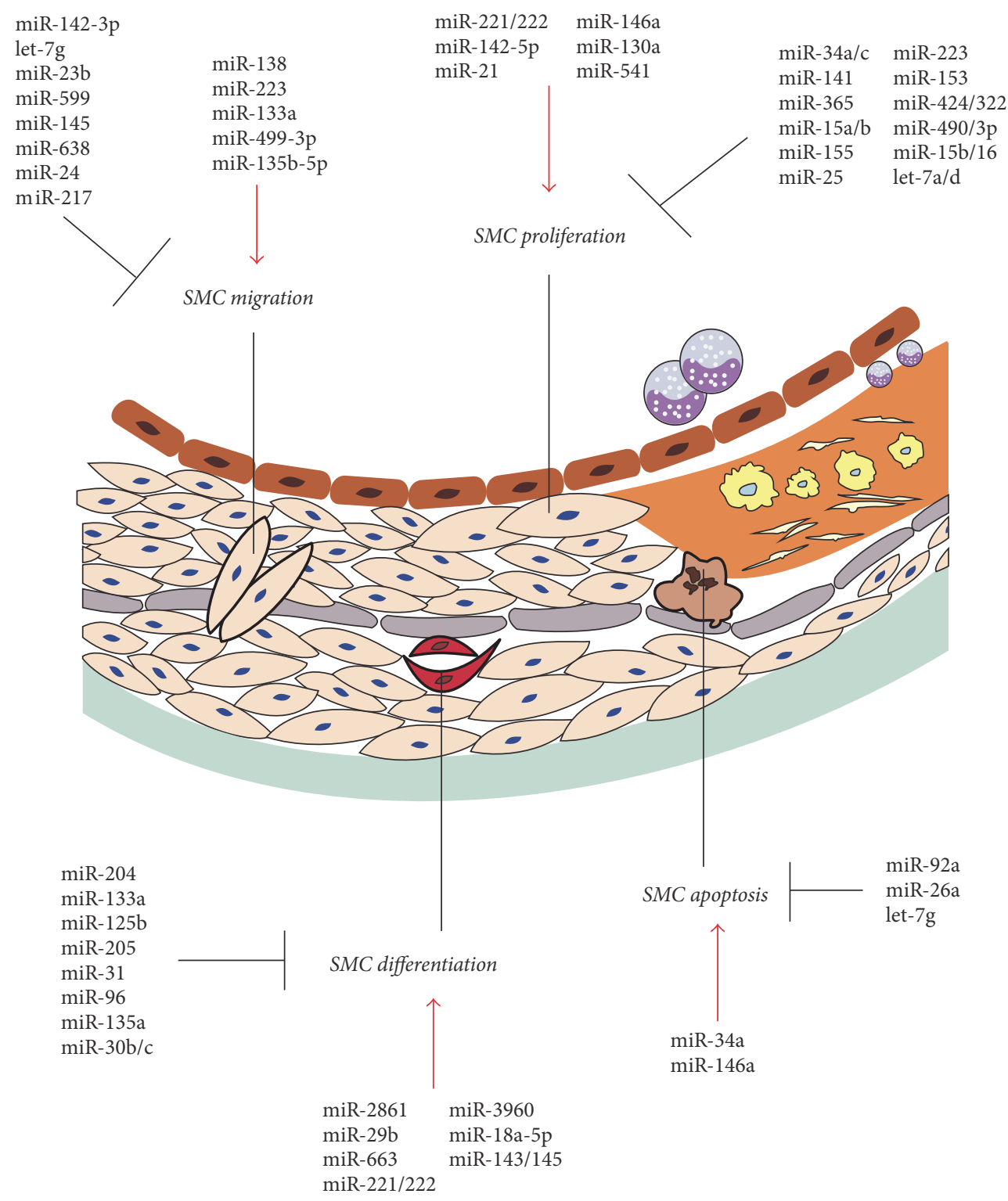

FIGURE 2: miRNAs that regulate phenotypic switching in VSMCs. The picture shows a variety of miRNAs that are involved in regulating the proliferation, migration, apoptosis, and differentiation of VSMCs. The red arrows indicate stimulatory effects, while the black arrows indicate inhibitory effects.

an efficient therapeutic approach to suppress atherosclerosis. In addition, other miRNAs, including miR-130a [96], miR135b-5p and miR-499a-3p [97], miR-142-5p [98], miR-223 [99], miR-155 [100], and miR-541 [101], could promote VSMC proliferation and migration by regulating their own target genes.

(2) miRNAs That Inhibit the Proliferation and Migration of VSMCs. Other miRNAs have been reported to inhibit the proliferation and migration of VSMCs. By preventing VSMC proliferation, neointimal progression in atherosclerosis may be controlled. The let-7 family plays an important role in
VSMC function. Let-7a decreased the proliferation of cultured VSMCs by reducing the expression of c-Myc and KRAS and could prevent intimal hyperplasia in an experimental vein graft model [102]. Overexpression of let-7d reduces VSMC growth by targeting KRAS [103]. Lower levels of let$7 \mathrm{~g}$ have been observed both in subjects with hypercholesterolemia and in mice fed a high-fat diet. The transfection of let-7g into VSMCs has been shown to significantly inhibit VSMCs proliferation and migration induced by ox-LDL by targeting LOX-1 [104]. Moreover, both miR-141 and miR490-3p could inhibit ox-LDL-induced VSMC proliferation through targeting of PAPP- $\alpha[105,106]$. 
Diabetes is another common age-related disease; VSMCs play a key role in the progress of diabetic atherosclerosis. miR-24 could inhibit high-glucose-induced VSMCs proliferation and migration by targeting high mobility group box1 (HMGB1) [107]. Meanwhile, the G1/S transition activated by platelet-derived growth factor-BB (PDGF-BB) could be blocked by miR-365 [108] and miR-15b [109]. Additionally, miR-638 also mediated inhibitory effects on PDGF-induced cell proliferation and migration in human aortic SMCs by targeting the NOR1/cyclin D pathway [110]. Qian et al. reported that upregulating miR-542-3p in old VSMCs could inhibit VSMCs proliferation by directly targeting spleen tyrosine kinase. This downregulation of miR-542-3p may explain agerelated neointimal hyperplasia in rats [111].

As mentioned in the miRNAs participate in the function of ECs, miR-34a and miR-34c inhibited VSMCs proliferation and migration through the modulation of Notch gene and stem cell factor (SCF) expression, respectively, which contributed to reducing neointimal hyperplasia $[75,112]$. Specifically, overexpression of miR-223 and miR-153 inhibited stretch stress-enhanced VSMCs proliferation via activation of the insulin-like growth factor-1 receptor and PI3KAKT signaling pathway [113]. Besides, miR-155 and miR217 would inhibit angiotensin II and homocysteine-induced VSMC proliferation and migration $[114,115]$. In contrast, miR-132 and miR-125b could block VSMC proliferation and neointimal hyperplasia in atherosclerosis [116, 117]. Other miRNAs that inhibit VSMCs proliferation and migration include miR-142-3p [118], miR-145 [119], miR-599 [120], miR25 [121], miR-23b [122], miR-15b/16 [123], and miR-29b [124].

2.2.3. miRNAs and VSMCs Differentiation and Calcification. Vascular calcification is a highly prevalent phenomenon among the elder population and is identified frequently in patients with atherosclerosis, diabetes mellitus, and chronic kidney disease (CKD) [125-127]. One component of the vascular calcification process involves the reprogramming and transdifferentiation of VSMCs to osteoblast-like cells [128-131]. These osteoblast-like SMCs generate and release calcifying matrix vesicles that are another essential factor involved in vascular calcification [132-134]. As the process of vascular calcification is tightly regulated and involves the genetic reprogramming of VSMCs, it is not surprising that there is accumulating evidence to support an integral role for miRNAs in this process [135-138].

The transdifferentiation of VSMCs to osteoblast-like cells that from the bone matrix is a recognized contributor to vascular calcification. Our previous studies had demonstrated that miR-204 and miR-133a could reduce osteoblastic differentiation of VSMCs induced by $\beta$-glycerophosphoric acid $(\beta-\mathrm{GP})$ via targeting runt-related transcription factor 2 (Runx2) $[9,10]$. Wen et al. also identified that overexpression of miR-125b could inhibit $\beta$-GP-induced osteogenic marker expression and the calcification of VSMCs. Moreover, miR$125 \mathrm{~b}$ targeted Etsl and regulated its protein expression in VSMCs [138]. Furthermore, endogenous miR-205 inhibits the differentiation of HA-VASMCs into osteoblast-like cells by targeting Runx 2 and Smad1, as evidenced by a decrease in ALP activity, osteocalcin secretion, and Runx2 expression
[139], whereas miR-2861 and miR-3960 in VSMCs enhance $\beta$-GP-induced osteogenic transdifferentiation of VSMCs by targeting histone deacetylase 5 or Homeobox A2, respectively, resulting in increased Runx2 protein production [140]. The overexpression of miR-29b promoted Pi-induced VSMC calcification; thus, it plays an important role in the progression of vascular calcification via osteoblastic differentiation in VSMCs [136].

Many other miRNAs could be important biomarkers of diseases through modulation of the VSMCs phenotype. CREG and VSMCs differentiation marker gene expression levels were shown to be suppressed by miR-31 [141]. BMP signaling downregulates the transcription of miR-96, which in turn leads to upregulation of Tribbles-like protein 3 (Trb3), an essential positive regulator of the BMP signaling pathway, and promotes the contractile phenotype in VSMCs [142]. When overexpression of miR-663 and miR-18a-5p promotes VSMCs differentiation markers, SM $\alpha$-actin and SM22 $\alpha$ are involved in VSMCs differentiation by targeting JunB/myosin light chain 9 and syndecan-4 expression, respectively $[143,144]$. mR145 acts to suppress TGF $\beta$-dependent ECM accumulation and fibrosis, while promoting TGF $\beta$-induced VSMCs differentiation [145]. At the same time, miR-145 and miR143 cooperatively target a network of transcription factors, including KLF4, myocardin, and ELK-1 (ELK1, member of the ETS oncogene family), to promote differentiation and repress the proliferation of SMC [146], and VSMC differentiation marker genes such as SM-actin, calponin, and SM-MHC are upregulated by premiR-145 and miR-145 mediated phenotypic modulation of VSMCs through its target gene KLF5 and its downstream signaling molecule, myocardin [147].

Other miRNAs are involved in modulating the differentiation of VSMCs. For example, miR-135a acts as a potential osteogenic differentiation suppressor in senescent VSMCs by targeting both KLF4 and STAT3 [137]. Increased calcium deposition was observed in the combined treatment with mimics of miR-221 and miR-222 [135]. In VSMCs, miR762, miR-714, and miR-712 were involved in calcification by disrupting Ca2t efflux proteins [148]. Additionally, BMP2 downregulates $\mathrm{miR}-30 \mathrm{~b}$ and $\mathrm{miR}-30 \mathrm{c}$ to increase Runx2 expression in VSMCs and promote mineralization and VSMCs calcification [149].

Several important miRNAs could regulate various functions of VSMCs. For instance, transfection of let-7g into VSMCs has been shown to significantly inhibit VSMCs proliferation and migration induced by ox-LDL by targeting LOX1 [104]. Moreover, let-7g can inhibit ox-LDL uptake and reduce apoptosis in SMCs via downregulation of cytochrome C [74]. In addition, miR-221/222 not only inhibits the differentiation of VSMCs but also promotes their proliferation and migration $[85,135]$. In the process of regulating VSMCs function, the SIRT1 gene also has important effects on VSMCs, as it does on ECs. For example, miR-34a can promote VSMCs apoptosis by modulating the expression of SIRT1 [76], while miR-138 can promote the proliferation and migration of VSMCs by inhibiting the expression of SIRT1 [94]. Just like the SIRT1 gene, KLF4, a member of the family of evolutionarily conserved zinc finger-containing transcription factors, could be taken as a regulatory target of different miRNAs 
to regulate the proliferation, migration and differentiation of VSMCs. For example, miR-146a could promote VSMC proliferation and migration by targeting KLF4 [91], whereas miR-15a acts as a direct transcriptional target of KLF4 that mediates the antiproliferative and antiangiogenic actions of VSMCs [150]. Meanwhile, miR-143 and miR-145 cooperatively target a network of KLF4 to promote differentiation and repress the proliferation of VSMCs [146].

\section{How to Analyze the Role of miRNAs in Cells}

The effects of miRNAs on the regulation of ECs and VSMCs are not a set of isolated processes; many miRNAs participate in modulating the function of both ECs and VSMCs including miR-221/222, miR-34a, miR-21, miR-217, miR-132, and the let-7 family. However, even the same miRNAs might have different effects on ECs and VSMCs. For example, miR-21 can enhance the rapamycin-induced inhibition of endothelial proliferation by targeting RhoB [37]. However, it can stimulate VSMCs proliferation and migration through suppression of c-Ski, and the low expression of miR-21 significantly inhibits cell proliferation and migration by targeting different genes $[88,89]$. miRNAs, however, could also have a similar influence on ECs and VSMCs. For instance, let$7 \mathrm{~g}$ negatively regulated apoptosis in the ECs by targeting caspase-3 expression [21]. Meanwhile, let-7g could inhibit SMC apoptosis by downregulating cytochrome C [74]. On the one hand, miR-221/222 could partly alleviate apoptotic cell death mediated by ox-LDL through the suppression of Ets-1 and its downstream target, p21 [18]. On the other hand, miR-221/222 also had antiapoptosis effects in VSMCs [85]. In addition, miR-34a expression is increased in senescent HUVECs and induces HUVEC senescence through the suppression of SIRT1 [29]; at the same time, it could inhibit cell proliferation and migration by targeting SIRT1 [39]. In VSMCs, miR-34a can also promote VSMCs senescence and inflammation through SIRT1 downregulation [76]. Furthermore, miR-34a inhibited VSMC proliferation and migration by modulating SCF expression [112]. The main explanation for the different roles of miRNA in ECs and VSMCs may be as follows: firstly, different cell types display their own unique characteristics and functions. Secondly, the particular structures and characteristics of different miRNAs play a key role in the functions of cells. Thirdly, the differing results may be related to the detailed conditions of the experiment. Finally, the target genes selected in the experiment may also influence miRNA functions. Different target genes have different biological properties; therefore, if miRNAs targeted the same genes in ECs and VSMCs, they will have similar effects. For example, miR-34a targets SIRT1 in both ECs and VSMCs; thus miR-34a has the same inhibitory effects on senescence and proliferation in the two cell types [29, 76, 112]. Nevertheless, different miRNAs with the same target genes may also produce different effects. For example, miR146 can downregulate SIRT1 and promote ECs senescence [30], whereas let-7g, also with SIRT1 as the target gene, has inhibitory effects on ECs senescence [33]. Therefore, it is necessary to assess the specific environment and the target genes when analyzing the role of a miRNA.

\section{Prospective Clinical Application of miRNAs as Diagnostic and Therapeutic Tool for Vascular Diseases}

miRNAs have become one of the most important gene regulators involved in almost all types of cellular processes, including vascular cell differentiation, migration, proliferation, senescence, and apoptosis. miRNAs that are detected in serum or plasma are collectively called circulating miRNAs and the source of that might be vesicles (exosomes and microparticles), proteins, or lipoprotein complexes, which might fulfill biological functions outside the cell and act as potential biomarkers for cardiovascular diseases [151]. Although various tissues such as the heart, lung, liver, and kidney contribute to the circulating miRNA pool, most of the miRNAs are derived from blood cells [152].

It is generally considered that circulating miRNAs may provide a specific signature that reflects a given disease state; thus, measurement of circulating miRNAs can serve as a diagnostic tool in cardiovascular disease. For example, $\mathrm{Li}$ et al. investigated the relative expression of miRNAs in intima samples of peripheral artery disease patients and found that miR-21, miR-27b, miR-130a, miR-210, and let-7f were significantly upregulated, whereas miR-221 and miR-22 were decreased. In addition, miR-27b, miR-210, and miR-130a were increased in serum samples. Such miRNAs would be regarded as biomarkers for early atherosclerosis [153]. Moreover, those miRNAs (miR-204, miR-125b, miR-205, and so on) that inhibit vascular calcification are downregulated, while other miRNAs (miR-2861, miR-390, and miR-29b) that could enhance vascular calcification are upregulated. Therefore, measuring circulating miRNAs levels might be a method to diagnose vascular calcification. So far, there are three major methods that could be applied for circulating miRNA identification and quantification. One is microarray technology, which has been utilized to provide a comprehensive miRNA expression profile. The other is real-time quantitative PCR (qRT-PCR), which is a simple tool that can efficiently determine the amount of a gene transcript in a given sample. The third one is next-generation sequencing (NGS), which provides us with an opportunity to examine all miRNA variants simultaneously, thereby helping in the identification of novel, disease-related miRNAs [154]. However, there are also some problems using these technologies to measure the circulating miRNAs. Firstly, the simplicity of this methodology can itself be problematic [155]. For example, there is no consensus as to whether plasma or serum is a more reliable substrate for measuring circulating miRNAs. Secondly, hemolysis during sample preparation, or even due to physiological processes, can also affect the levels of circulating miRNAs [156]. Moreover, antiplatelet treatment may affect circulating miRNAs in plasma and serum samples and may act as a confounding factor in case-control studies relating plasma miRNAs to cardiovascular disease [157]. Finally, different tissues could express the same miRNAs, which are all transmitted to the blood; thus, the measurement of circulating miRNAs lacks specificity. Therefore, there is a long way to go to increase the diagnostic accuracy of circulating miRNAs to diagnose cardiovascular diseases. 
Specific miRNA expression can be modulated by genetic approaches including overexpression or silencing of the prospective miRNA [158]. Thus, delivery of miRNA mimics into the proper tissue can provide a therapeutic benefit by enhancing the levels of specific miRNAs whose expression is downregulated in the disease state. Chen et al. demonstrated that overexpression of miR-126 inhibits vascular ECs apoptosis through targeting of PI3K/Akt signaling [15]. Consistent with this study, adenovirus-mediated restoration of miR-145 into rat balloon-injured carotid arteries in vivo significantly inhibited neointimal lesion formation [147]. Nevertheless, for specific miRNAs that are upregulated during disease, silencing of specific miRNAs would be beneficial. Currently, modified oligonucleotides can be designed to complement either the mature miRNA or its precursors leading to the inhibition of specific miRNA [159]. Liu et al. have applied modified antisense oligonucleotides to successfully knock down miR221 and miR-222 in cultured VSMCs and significantly inhibit cell proliferation and neointimal growth in rat ballooninjured carotid arteries [85]. However, because miRNAs are endogenous, restoration of aberrantly expressed miRNAs, both upregulated and downregulated, to physiological levels cannot be achieved without some unexpected side effects. For instance, the inhibition of a specific miRNA may be beneficial concerning atherosclerosis progression but may adversely affect other organ systems causing immunosuppression, liver damage, or even oncogenesis.

It is well known that miRNAs have an inhibitory effect in their targets mRNA transcription and consequently, on gene expression. In other words, the inhibition of miRNAs induces gene expression while the addition or enhancement of miRNAs has the opposite effect. Hence, the greatest challenge here lies in the ability to predict the exact effects of miRNA modulation in the human body. However, one miRNA can have multiple targets; for example, miR-21 can enhance the rapamycin-induced inhibition of endothelial proliferation by targeting RhoB [37]. Meanwhile, miR-21 significantly inhibited VSMC proliferation and migration by targeting tropomyosin and AP-1 $[88,89]$. One gene can also be regulated by several miRNAs. For instance, miR217 and miR-146a promote senescence with a reduction of SIRT1 in ECs [30,31], whereas let-7g has an effect on reducing ECs senescence by increasing the SIRT1 protein [33]. Keeping this in mind, miRNA-based therapy may have both advantages and disadvantages. miRNAs that have only a single target gene should be easy to suppress using anti-miRNA technology, which represents an advantage. However, the suppression of miRNAs that have multiple target genes will affect several genes and might induce some unexpected side effects, which could be a disadvantage [160]. Therefore, although targeting miRNAs represents promising therapeutic strategies, careful monitoring and studying of these interactions is essential in order to guarantee a safe application in humans.

\section{Conclusion}

Aging and its associated diseases remain a huge burden especially within the next decades; research efforts are increasing to identify the underlying molecular mechanisms and especially innovative treatment approaches to diseases closely associated with aging. To date, accumulating evidence has revealed that miRNAs are becoming one of the most fascinating areas of biology and play a crucial role in regulating aging processes in animal models and humans. The relative role of different miRNAs in vascular biology as direct or indirect posttranscriptional regulators of genes implicated in structural remodeling, inflammation, angiogenesis, atherosclerosis, in-stent restenosis, and thrombosis indicates that miRNAs may serve as promising drug targets or potential biomarkers in the prevention and management of vascular disorders. In this review, we have summarized the roles of miRNAs in the regulation of vascular aging, especially with respect to EC and VSMC functions, including differentiation, proliferation, migration, senescence, and apoptosis, all of which play critical roles in the pathogenesis of vascular aging. With rigorous fundamental and clinical studies, a clearer understanding of miRNAs as biomarkers and targets for cardiovascular disease will provide new insight into vascular aging and aging-related diseases.

\section{Competing Interests}

The authors declare that there is no conflict of interests regarding the publication of this paper.

\section{Acknowledgments}

This work was supported by the National Basic Research Program of China (no. 2014CB910500) and the National Natural Science Foundation of China (no. 81370931 and no. 81501212). The authors acknowledge PRS for helping in editing and proofreading the English of the manuscript.

\section{References}

[1] "Chronic disease management in ageing populations," The Lancet, vol. 379, no. 9829, p. 1851, 2012.

[2] B. G. Childs, M. Durik, D. J. Baker, and J. M. Van Deursen, "Cellular senescence in aging and age-related disease: from mechanisms to therapy," Nature Medicine, vol. 21, no. 12, pp. 14241435, 2015.

[3] Y.-J. Wang, Y. Wang, J.-K. Zhan et al., "Sarco-osteoporosis: prevalence and association with frailty in Chinese communitydwelling older adults," International Journal of Endocrinology, vol. 2015, Article ID 482940, 2015.

[4] A. Eckers and J. Haendeler, "Endothelial cells in health and disease," Antioxidants \& Redox Signaling, vol. 22, no. 14, pp. 12091211, 2015.

[5] B. A. Monk and S. J. George, "The effect of ageing on vascular smooth muscle cell behaviour-a mini-review," Gerontology, vol. 61, no. 5, pp. 416-426, 2015.

[6] T. Boettger, N. Beetz, S. Kostin et al., "Acquisition of the contractile phenotype by murine arterial smooth muscle cells depends on the Mir143/145 gene cluster," The Journal of Clinical Investigation, vol. 119, no. 9, pp. 2634-2647, 2009.

[7] Z. Ungvari, G. Kaley, R. De Cabo, W. E. Sonntag, and A. Csiszar, "Mechanisms of vascular aging: new perspectives," Journals of Gerontology-Series A Biological Sciences and Medical Sciences, vol. 65, no. 10, pp. 1028-1041, 2010. 
[8] D. P. Bartel, "MicroRNAs: genomics, biogenesis, mechanism, and function," Cell, vol. 116, no. 2, pp. 281-297, 2004.

[9] R.-R. Cui, S.-J. Li, L.-J. Liu et al., "MicroRNA-204 regulates vascular smooth muscle cell calcification in vitro and in vivo," Cardiovascular Research, vol. 96, no. 2, pp. 320-329, 2012.

[10] X.-B. Liao, Z.-Y. Zhang, K. Yuan et al., "MiR-133a modulates osteogenic differentiation of vascular smooth muscle cells," Endocrinology, vol. 154, no. 9, pp. 3344-3352, 2013.

[11] R. Menghini, R. Stöhr, and M. Federici, "MicroRNAs in vascular aging and atherosclerosis," Ageing Research Reviews, vol. 17, pp. 68-78, 2014.

[12] S. Lee, E. Choi, M. J. Cha, A. J. Park, C. Yoon, and K. C. Hwang, "Impact of miRNAs on cardiovascular aging," Journal of Geriatric Cardiology, vol. 12, no. 5, pp. 569-574, 2015.

[13] M. Weber, M. B. Baker, J. P. Moore, and C. D. Searles, "MiR21 is induced in endothelial cells by shear stress and modulates apoptosis and eNOS activity," Biochemical and Biophysical Research Communications, vol. 393, no. 4, pp. 643-648, 2010.

[14] Y. Zhang, W. Qin, L. Zhang et al., "MicroRNA-26a prevents endothelial cell apoptosis by directly targeting TRPC6 in the setting of atherosclerosis," Scientific Reports, vol. 5, article 9401, 2015.

[15] L. Chen, J. Wang, B. Wang et al., "MiR-126 inhibits vascular endothelial cell apoptosis through targeting PI3K/Akt signaling," Annals of Hematology, vol. 95, no. 3, pp. 365-374, 2016.

[16] M. Ye, D. Li, J. Yang et al., "MicroRNA-130a targets MAP3K12 to modulate diabetic endothelial progenitor cell function," Cellular Physiology and Biochemistry, vol. 36, no. 2, pp. 712-726, 2015.

[17] H. J. Lee, E. J. Lee, and M. Seo, "Galphal2 protects vascular endothelial cells from serum withdrawal-induced apoptosis through regulation of miR-155," Yonsei Medical Journal, vol. 57, no. 1, pp. 247-253, 2016.

[18] B. Qin, Y. Cao, H. Yang, B. Xiao, and Z. Lu, "MicroRNA-221/222 regulate ox-LDL-induced endothelial apoptosis via Ets-1/p21 inhibition," Molecular and Cellular Biochemistry, vol. 405, no. 1-2, pp. 115-124, 2015.

[19] D. Liu, X.-L. Zhang, C.-H. Yan et al., "MicroRNA-495 regulates the proliferation and apoptosis of human umbilical vein endothelial cells by targeting chemokine CCL2," Thrombosis Research, vol. 135, no. 1, pp. 146-154, 2015.

[20] M.-H. Bao, Y.-W. Zhang, X.-Y. Lou, Y. Cheng, and H.-H. Zhou, "Protective effects of Let-7a and Let-7b on oxidized low-density lipoprotein induced endothelial cell injuries," PLoS ONE, vol. 9, no. 9, Article ID e106540, 2014.

[21] Y. Zhang, N. Chen, J. Zhang, and Y. Tong, "Hsa-Let-7g miRNA targets caspase- 3 and inhibits the apoptosis induced by ox-LDL in endothelial cells," International Journal of Molecular Sciences, vol. 14, no. 11, pp. 22708-22720, 2013.

[22] Y. Tang, Y. C. Zhang, Y. Chen, Y. Xiang, C. X. Shen, and Y. G. Li, "The role of miR-19b in the inhibition of endothelial cell apoptosis and its relationship with coronary artery disease," Scientific Reports, vol. 5, article 15132, 2015.

[23] L. Zhang, D. Huang, Q. Wang et al., "MiR-132 inhibits expression of SIRT1 and induces pro-inflammatory processes of vascular endothelial inflammation through blockade of the SREBP-1c metabolic pathway," Cardiovascular Drugs and Therapy, vol. 28, no. 4, pp. 303-311, 2014.

[24] A. Magenta, C. Cencioni, P. Fasanaro et al., "MiR-200c is upregulated by oxidative stress and induces endothelial cell apoptosis and senescence via ZEB1 inhibition," Cell Death and Differentiation, vol. 18, no. 10, pp. 1628-1639, 2011.
[25] Y. Pan, H. Liang, H. Liu et al., "Platelet-secreted MicroRNA223 promotes endothelial cell apoptosis induced by advanced glycation end products via targeting the insulin-like growth factor 1 receptor," Journal of Immunology, vol. 192, no. 1, pp. 437446, 2014.

[26] B. Qin, B. Xiao, D. Liang, J. Xia, Y. Li, and H. Yang, "MicroRNAs expression in ox-LDL treated HUVECs: MiR-365 modulates apoptosis and Bcl-2 expression," Biochemical and Biophysical Research Communications, vol. 410, no. 1, pp. 127-133, 2011.

[27] J. Fan, W. Zhang, and Q. Liu, "Human cytomegalovirus-encoded miR-US25-1 aggravates the oxidised low density lipoproteininduced apoptosis of endothelial cells," BioMed Research International, vol. 2014, Article ID 531979, 13 pages, 2014.

[28] E. Takeda, Y. Suzuki, and Y. Sato, "Age-associated downregulation of vasohibin-1 in vascular endothelial cells," Aging Cell, vol. 15, no. 5, pp. 885-892, 2016.

[29] T. Ito, S. Yagi, and M. Yamakuchi, "MicroRNA-34a regulation of endothelial senescence," Biochemical and Biophysical Research Communications, vol. 398, no. 4, pp. 735-740, 2010.

[30] M. Vasa-Nicotera, H. Chen, P. Tucci et al., "miR-146a is modulated in human endothelial cell with aging," Atherosclerosis, vol. 217, no. 2, pp. 326-330, 2011.

[31] R. Menghini, V. Casagrande, M. Cardellini et al., "MicroRNA 217 modulates endothelial cell senescence via silent information regulator 1," Circulation, vol. 120, no. 15, pp. 1524-1532, 2009.

[32] C. Rippe, M. Blimline, K. A. Magerko et al., "MicroRNA changes in human arterial endothelial cells with senescence: relation to apoptosis, eNOS and inflammation," Experimental Gerontology, vol. 47, no. 1, pp. 45-51, 2012.

[33] Y.-C. Liao, Y.-S. Wang, Y.-C. Guo, W.-L. Lin, M.-H. Chang, and S.-H. H. Juo, "Let-7g improves multiple endothelial functions through targeting transforming growth factor-beta and sirt-1 signaling," Journal of the American College of Cardiology, vol. 63, no. 16, pp. 1685-1694, 2014.

[34] Z. Yang, L. Wu, X. Zhu et al., "MiR-29a modulates the angiogenic properties of human endothelial cells," Biochemical and Biophysical Research Communications, vol. 434, no. 1, pp. 143149,2013

[35] A. Schober, M. Nazari-Jahantigh, Y. Wei et al., "MicroRNA-126$5 \mathrm{p}$ promotes endothelial proliferation and limits atherosclerosis by suppressing Dlk1," Nature Medicine, vol. 20, no. 4, pp. 368376, 2014.

[36] N. Feng, Z. Wang, Z. Zhang, X. He, C. Wang, and L. Zhang, "miR-487b promotes human umbilical vein endothelial cell proliferation, migration, invasion and tube formation through regulating THBS1," Neuroscience Letters, vol. 591, pp. 1-7, 2015.

[37] C. Jin, Y. Zhao, L. Yu, S. Xu, and G. Fu, "MicroRNA-21 mediates the rapamycin-induced suppression of endothelial proliferation and migration," FEBS Letters, vol. 587, no. 4, pp. 378-385, 2013.

[38] W. Zhang, L. Yan, Y. Li et al., "Roles of miRNA-24 in regulating endothelial nitric oxide synthase expression and vascular endothelial cell proliferation," Molecular and Cellular Biochemistry, vol. 405, no. 1-2, pp. 281-289, 2015.

[39] C. Iaconetti, A. Polimeni, S. Sorrentino et al., "Inhibition of miR-92a increases endothelial proliferation and migration in vitro as well as reduces neointimal proliferation in vivo after vascular injury," Basic Research in Cardiology, vol. 107, no. 5, article 296, 2012.

[40] K. Chen, W. Fan, X. Wang, X. Ke, G. Wu, and C. Hu, "MicroRNA-101 mediates the suppressive effect of laminar shear stress on mTOR expression in vascular endothelial cells," 
Biochemical and Biophysical Research Communications, vol. 427, no. 1, pp. 138-142, 2012.

[41] D. Svensson, O. Gidlöf, K. M. Turczyńska, D. Erlinge, S. Albinsson, and B.-O. Nilsson, "Inhibition of MicroRNA-125a promotes human endothelial cell proliferation and viability through an antiapoptotic mechanism," Journal of Vascular Research, vol. 51, no. 3, pp. 239-245, 2014.

[42] L. Zhang, M. Zhou, G. Qin, N. L. Weintraub, and Y. Tang, "MiR92a regulates viability and angiogenesis of endothelial cells under oxidative stress," Biochemical and Biophysical Research Communications, vol. 446, no. 4, pp. 952-958, 2014.

[43] S. Wang, A. B. Aurora, B. A. Johnson et al., "The endothelialspecific microRNA miR-126 governs vascular integrity and angiogenesis," Developmental Cell, vol. 15, no. 2, pp. 261-271, 2008.

[44] K.-J. Yin, K. Olsen, M. Hamblin, J. Zhang, S. P. Schwendeman, and Y. E. Chen, "Vascular endothelial cell-specific microRNA15a inhibits angiogenesis in hindlimb ischemia," The Journal of Biological Chemistry, vol. 287, no. 32, pp. 27055-27064, 2012.

[45] A.-L. Pin, F. Houle, M. Guillonneau, É. R. Paquet, M. J. Simard, and J. Huot, "MIR-20a represses endothelial cell migration by targeting MKK3 and inhibiting p38 MAP kinase activation in response to VEGF," Angiogenesis, vol. 15, no. 4, pp. 593-608, 2012.

[46] C. Sabatel, L. Malvaux, N. Bovy et al., "MicroRNA-21 exhibits antiangiogenic function by targeting RhoB expression in endothelial cells," PLoS ONE, vol. 6, no. 2, Article ID e16979, 2011.

[47] Y. Zhang, Y. Liu, H. Zhang, M. Wang, and J. Zhang, "MmuMIR-351 attenuates the survival of cardiac arterial endothelial cells through targeting STAT3 in the atherosclerotic mice," Biochemical and Biophysical Research Communications, vol. 468, no. 1-2, pp. 300-305, 2015.

[48] Q. Duan, L. Yang, W. Gong et al., "MicroRNA-214 is upregulated in heart failure patients and suppresses XBP1-mediated endothelial cells angiogenesis," Journal of Cellular Physiology, vol. 230, no. 8, pp. 1964-1973, 2015.

[49] L. Shi, B. Fisslthaler, N. Zippel et al., "MicroRNA-223 antagonizes angiogenesis by targeting $\beta 1$ integrin and preventing growth factor signaling in endothelial cells," Circulation Research, vol. 113, no. 12, pp. 1320-1330, 2013.

[50] L. Poliseno, A. Tuccoli, L. Mariani et al., "MicroRNAs modulate the angiogenic properties of HUVECs," Blood, vol. 108, no. 9, pp. 3068-3071, 2006.

[51] A. Maimaiti, A. Maimaiti, Y. Yang, and Y. Ma, "MiR-106b exhibits an anti-angiogenic function by inhibiting STAT3 expression in endothelial cells," Lipids in Health and Disease, vol. 15, article 51, 2016.

[52] J. Zhou, K.-C. Wang, W. Wu et al., "MicroRNA-21 targets peroxisome proliferators-activated receptor- $\alpha$ in an autoregulatory loop to modulate flow-induced endothelial inflammation," Proceedings of the National Academy of Sciences of the United States of America, vol. 108, no. 25, pp. 10355-10360, 2011.

[53] X. Loyer, S. Potteaux, A.-C. Vion et al., "Inhibition of microRNA-92a prevents endothelial dysfunction and atherosclerosis in mice," Circulation Research, vol. 114, no. 3, pp. 434-443, 2014.

[54] S. Demolli, C. Doebele, A. Doddaballapur et al., "MicroRNA-30 mediates anti-inflammatory effects of shear stress and KLF2 via repression of angiopoietin 2," Journal of Molecular and Cellular Cardiology, vol. 88, pp. 111-119, 2015.
[55] T. A. Harris, M. Yamakuchi, M. Ferlito, J. T. Mendell, and C. J. Lowenstein, "microRNA-126 regulates endothelial expression of vascular cell adhesion molecule 1," Proceedings of the National Academy of Sciences of the United States of America, vol. 105, no. 5, pp. 1516-1521, 2008.

[56] T. Liu, D. Shen, S. Xing et al., "Attenuation of exogenous angiotensin II stress-induced damage and apoptosis in human vascular endothelial cells via microRNA-155 expression," International Journal of Molecular Medicine, vol. 31, no. 1, pp. 188-196, 2013.

[57] X. Sun, S. He, A. K. M. Wara et al., "Systemic delivery of MicroRNA-181b inhibits nuclear factor- $\kappa \mathrm{B}$ activation, vascular inflammation, and atherosclerosis in apolipoprotein E-deficient mice," Circulation Research, vol. 114, no. 1, pp. 32-40, 2014.

[58] C.-W. Ni, H. Qiu, and H. Jo, "MicroRNA-663 upregulated by oscillatory shear stress plays a role in inflammatory response of endothelial cells," American Journal of Physiology-Heart and Circulatory Physiology, vol. 300, no. 5, pp. H1762-H1769, 2011.

[59] S. Dimmeler and P. Nicotera, "MicroRNAs in age-related diseases," EMBO Molecular Medicine, vol. 5, no. 2, pp. 180-190, 2013.

[60] F.-J. Liu, T. Wen, and L. Liu, "MicroRNAs as a novel cellular senescence regulator," Ageing Research Reviews, vol. 11, no. 1, pp. 41-50, 2012.

[61] T. Staszel, B. Zapała, A. Polus et al., "Role of microRNAs in endothelial cell pathophysiology," Polskie Archiwum Medycyny Wewnetrznej, vol. 121, no. 10, pp. 361-367, 2011.

[62] N. Peng, N. Meng, S. Wang et al., "An activator of mTOR inhibits oxLDL-induced autophagy and apoptosis in vascular endothelial cells and restricts atherosclerosis in apolipoprotein $\mathrm{E}^{-/-}$mice," Scientific Reports, vol. 4, article 5519, 2014.

[63] A. Zernecke, K. Bidzhekov, H. Noels et al., "Delivery of microRNA-126 by apoptotic bodies induces CXCL12-dependent vascular protection," Science Signaling, vol. 2, no. 100, article ra81, 2009.

[64] T. Minamino and I. Komuro, "Vascular cell senescence: contribution to atherosclerosis," Circulation Research, vol. 100, no. 1, pp. 15-26, 2007.

[65] J. Lahteenvuo and A. Rosenzweig, "Effects of aging on angiogenesis," Circulation Research, vol. 110, no. 9, pp. 1252-1264, 2012.

[66] C. Doebele, A. Bonauer, A. Fischer et al., "Members of the microRNA-17-92 cluster exhibit a cell-intrinsic antiangiogenic function in endothelial cells," Blood, vol. 115, no. 23, pp. 49444950, 2010

[67] A. Bonauer, G. Carmona, M. Iwasaki et al., "MicroRNA-92a controls angiogenesis and functional recovery of ischemic tissues in Mice," Science, vol. 324, no. 5935, pp. 1710-1713, 2009.

[68] M. Soufi-zomorrod, A. Hajifathali, F. Kouhkan, M. Mehdizadeh, S. M. A. H. Rad, and M. Soleimani, "MicroRNAs modulating angiogenesis: miR-129-1 and miR-133 act as angio-miR in HUVECs," Tumor Biology, vol. 37, no. 7, pp. 9527-9534, 2016.

[69] Y. Hu, F. Deng, J. Song et al., "Evaluation of miR-29c inhibits endotheliocyte migration and angiogenesis of human endothelial cells by suppressing the insulin like growth factor 1," American Journal of Translational Research, vol. 7, no. 5, pp. 866-877, 2015.

[70] I. Pantsulaia, W. M. Ciszewski, and J. Niewiarowska, "Senescent endothelial cells: potential modulators of immunosenescence and ageing," Ageing Research Reviews, vol. 29, pp. 13-25, 2016. 
[71] J. Wu, S. Xia, B. Kalionis, W. Wan, and T. Sun, "The role of oxidative stress and inflammation in cardiovascular aging," BioMed Research International, vol. 2014, Article ID 615312, 13 pages, 2014.

[72] B. Qin, H. Yang, and B. Xiao, "Role of microRNAs in endothelial inflammation and senescence," Molecular Biology Reports, vol. 39, no. 4, pp. 4509-4518, 2012.

[73] L. Maegdefessel, K. J. Rayner, and N. J. Leeper, "MicroRNA regulation of vascular smooth muscle function and phenotype: early career committee contribution," Arteriosclerosis, Thrombosis, and Vascular Biology, vol. 35, no. 1, pp. 2-6, 2015.

[74] Z. Ding, X. Wang, M. Khaidakov, S. Liu, and J. L. Mehta, "MicroRNA hsa-let-7g targets lectin-like oxidized low-density lipoprotein receptor-1 expression and inhibits apoptosis in human smooth muscle cells," Experimental Biology and Medicine, vol. 237, no. 9, pp. 1093-1100, 2012.

[75] N. Choe, J.-S. Kwon, Y. S. Kim et al., "The microRNA miR-34c inhibits vascular smooth muscle cell proliferation and neointimal hyperplasia by targeting stem cell factor," Cellular Signalling, vol. 27, no. 6, pp. 1056-1065, 2015.

[76] I. Badi, I. Burba, C. Ruggeri et al., "MicroRNA-34a induces vascular smooth muscle cells senescence by SIRT1 downregulation and promotes the expression of age-associated pro-inflammatory secretory factors," Journals of Gerontology. A Biological Sciences and Medical Sciences, vol. 70, no. 11, pp. 1304-1311, 2015.

[77] J. M. Boucher, S. M. Peterson, S. Urs, C. Zhang, and L. Liaw, “The miR-143/145 cluster is a novel transcriptional target of Jagged1/Notch signaling in vascular smooth muscle cells," The Journal of Biological Chemistry, vol. 286, no. 32, pp. 28312-28321, 2011.

[78] M. C. H. Clarke, N. Figg, J. J. Maguire et al., "Apoptosis of vascular smooth muscle cells induces features of plaque vulnerability in atherosclerosis," Nature Medicine, vol. 12, no. 9, pp. 10751080, 2006.

[79] P. Tan, Y. J. Wang, S. Li et al., "The PI3K/Akt/mTOR pathway regulates the replicative senescence of human VSMCs," Molecular and Cellular Biochemistry, vol. 422, no. 1-2, pp. 1-10, 2016.

[80] Z. Ding, X. Wang, L. Schnackenberg et al., "Regulation of autophagy and apoptosis in response to ox-LDL in vascular smooth muscle cells, and the modulatory effects of the microRNA hsa-let-7g," International Journal of Cardiology, vol. 168, no. 2, pp. 1378-1385, 2013.

[81] L. Zhang, M. Zhou, Y. Wang et al., "miR-92a inhibits vascular smooth muscle cell apoptosis: role of the MKK4-JNK pathway," Apoptosis, vol. 19, no. 6, pp. 975-983, 2014.

[82] Z. W. Wu, Y. F. Liu, S. Wang, and B. Li, "miRNA-146a induces vascular smooth muscle cell apoptosis in a rat model of coronary heart disease via NF- $\kappa$ B pathway," Genetics and Molecular Research, vol. 14, no. 4, pp. 18703-18712, 2015.

[83] J. Li, L. Zhao, X. He, T. Yang, and K. Yang, "MiR-21 inhibits c-Ski signaling to promote the proliferation of rat vascular smooth muscle cells," Cellular Signalling, vol. 26, no. 4, pp. 724-729, 2014.

[84] S. Dong, W. Xiong, J. Yuan, J. Li, J. Liu, and X. Xu, "MiRNA146a regulates the maturation and differentiation of vascular smooth muscle cells by targeting NF- $\kappa$ B expression," Molecular Medicine Reports, vol. 8, no. 2, pp. 407-412, 2013.

[85] X. Liu, Y. Cheng, S. Zhang, Y. Lin, J. Yang, and C. Zhang, "A necessary role of miR-221 and miR-222 in vascular smooth muscle cell proliferation and neointimal hyperplasia," Circulation Research, vol. 104, no. 4, pp. 476-487, 2009.

[86] J. J. Stein, C. Iwuchukwu, K. G. Maier, and V. Gahtan, "Thrombospondin-1-induced vascular smooth muscle cell migration and proliferation are functionally dependent on microRNA-21," Surgery, vol. 155, no. 2, pp. 228-233, 2014.

[87] J. Li, P. Li, Y. Zhang et al., "C-Ski inhibits the proliferation of vascular smooth muscle cells via suppressing Smad3 signaling but stimulating p38 pathway," Cellular Signalling, vol. 25, no. 1, pp. 159-167, 2013.

[88] M. Wang, W. Li, G.-Q. Chang et al., "MicroRNA-21 regulates vascular smooth muscle cell function via targeting tropomyosin 1 in arteriosclerosis obliterans of lower extremities," Arteriosclerosis, Thrombosis, and Vascular Biology, vol. 31, no. 9, pp. 20442053, 2011.

[89] Y. Li, L. Yan, W. Zhang et al., "MicroRNA-21 inhibits plateletderived growth factor-induced human aortic vascular smooth muscle cell proliferation and migration through targeting activator protein-1," American Journal of Translational Research, vol. 6, no. 5, pp. 507-516, 2014.

[90] H. Wang, M. Jiang, Z. Xu et al., "miR-146b-5p promotes VSMC proliferation and migration," International Journal of Clinical and Experimental Pathology, vol. 8, no. 10, pp. 12901-12907, 2015.

[91] S.-G. Sun, B. Zheng, M. Han et al., "MiR-146a and Krüppel-like factor 4 form a feedback loop to participate in vascular smooth muscle cell proliferation," EMBO Reports, vol. 12, no. 1, pp. 5662, 2011.

[92] J. Cao, K. Zhang, J. Zheng, and R. Dong, "MicroRNA-146a and -21 cooperate to regulate vascular smooth muscle cell proliferation via modulation of the Notch signaling pathway," Molecular Medicine Reports, vol. 11, no. 4, pp. 2889-2895, 2015.

[93] X. Liu, Y. Cheng, J. Yang, L. Xu, and C. Zhang, "Cell-specific effects of miR-221/222 in vessels: molecular mechanism and therapeutic application," Journal of Molecular and Cellular Cardiology, vol. 52, no. 1, pp. 245-255, 2012.

[94] J. Xu, L. Li, H.-F. Yun, and Y.-S. Han, "MiR-138 promotes smooth muscle cells proliferation and migration in $\mathrm{db} / \mathrm{db}$ mice through down-regulation of SIRT1," Biochemical and Biophysical Research Communications, vol. 463, no. 4, pp. 1159-1164, 2015.

[95] S. Gao, M. Wassler, L. Zhang et al., "MicroRNA-133a regulates insulin-like growth factor-1 receptor expression and vascular smooth muscle cell proliferation in murine atherosclerosis," Atherosclerosis, vol. 232, no. 1, pp. 171-179, 2014.

[96] W.-H. Wu, C.-P. Hu, X.-P. Chen et al., "MicroRNA-130a mediates proliferation of vascular smooth muscle cells in hypertension," American Journal of Hypertension, vol. 24, no. 10, pp.10871093, 2011.

[97] Z. Xu, Y. Han, J. Liu et al., "MiR-135b-5p and MiR-499a-3p promote cell proliferation and migration in atherosclerosis by directly targeting MEF2C," Scientific Reports, vol. 5, Article ID 12276, 2015.

[98] H. J. Kee, S. Park, J.-S. Kwon et al., "B cell translocation gene, a direct target of miR-142-5p, inhibits vascular smooth muscle cell proliferation by down-regulating cell cycle progression," FEBS Letters, vol. 587, no. 15, pp. 2385-2392, 2013.

[99] A. Y. Rangrez, E. M’Baya-Moutoula, V. Metzinger-Le Meuth et al., "Inorganic phosphate accelerates the migration of vascular smooth muscle cells: evidence for the involvement of miR-223," PLoS ONE, vol. 7, no. 10, Article ID e47807, 2012.

[100] J. Zhang, F. Zhao, X. Yu, X. Lu, and G. Zheng, "MicroRNA-155 modulates the proliferation of vascular smooth muscle cells by targeting endothelial nitric oxide synthase," International Journal of Molecular Medicine, vol. 35, no. 6, pp. 1708-1714, 2015.

[101] F. Yang, Z. Xu, S. Duan, and M. Luo, "MicroRNA-541 promotes the proliferation of vascular smooth muscle cells by targeting 
IRF7," American Journal of Translational Research, vol. 8, no. 2, pp. 506-515, 2016.

[102] H. Cao, X. Hu, Q. Zhang et al., "Upregulation of let-7a inhibits vascular smooth muscle cell proliferation in vitro and in vein graft intimal hyperplasia in rats," Journal of Surgical Research, vol. 192, no. 1, pp. 223-233, 2014.

[103] M.-L. Yu, J.-F. Wang, G.-K. Wang et al., "Vascular smooth muscle cell proliferation is influenced by let-7d microRNA and its interaction with KRAS," Circulation Journal, vol. 75, no. 3, pp. 703-709, 2011.

[104] K.-C. Chen, I.-C. Hsieh, E. Hsi et al., "Negative feedback regulation between microRNA let-7g and the oxLDL receptor LOX1," Journal of Cell Science, vol. 124, no. 23, pp. 4115-4124, 2011.

[105] Y. Zhang, B. Chen, L. Ming et al., "MicroRNA-141 inhibits vascular smooth muscle cell proliferation through targeting PAPPA," International Journal of Clinical and Experimental Pathology, vol. 8, no. 11, pp. 14401-14408, 2015.

[106] Y. Sun, D. Chen, L. Cao et al., "MiR-490-3p modulates the proliferation of vascular smooth muscle cells induced by ox-LDL through targeting PAPP-A," Cardiovascular Research, vol. 100, no. 2, pp. 272-279, 2013.

[107] J. Yang, L. Chen, J. Ding et al., "MicroRNA-24 inhibits high glucose-induced vascular smooth muscle cell proliferation and migration by targeting HMGB1," Gene, vol. 586, no. 2, pp. 268273, 2016.

[108] P. Zhang, C. Zheng, H. Ye et al., "MicroRNA-365 inhibits vascular smooth muscle cell proliferation through targeting cyclin D1," International Journal of Medical Sciences, vol. 11, no. 8, pp. 765-770, 2014.

[109] S. Kim and H. Kang, "miR-15b induced by platelet-derived growth factor signaling is required for vascular smooth muscle cell proliferation," BMB Reports, vol. 46, no. 11, pp. 550-554, 2013.

[110] P. Li, Y. Liu, B. Yi et al., "MicroRNA-638 is highly expressed in human vascular smooth muscle cells and inhibits PDGFBB-induced cell proliferation and migration through targeting orphan nuclear receptor NOR1," Cardiovascular Research, vol. 99, no. 1, pp. 185-193, 2013.

[111] D.-H. Qian, P. Gao, H. Feng, Z.-X. Qin, J.-B. Li, and L. Huang, "Down-regulation of mir-542-3p promotes neointimal formation in the aging rat," Vascular Pharmacology, vol. 72, pp. 118129, 2015.

[112] Q. Chen, F. Yang, M. Guo et al., "miRNA-34a reduces neointima formation through inhibiting smooth muscle cell proliferation and migration," Journal of Molecular and Cellular Cardiology, vol. 89, pp. 75-86, 2015.

[113] L. Song, P. Duan, P. Guo et al., "Downregulation of miR-223 and miR-153 mediates mechanical stretch-stimulated proliferation of venous smooth muscle cells via activation of the insulinlike growth factor-1 receptor," Archives of Biochemistry and Biophysics, vol. 528, no. 2, pp. 204-211, 2012.

[114] L.-X. Yang, G. Liu, G.-F. Zhu et al., "MicroRNA-155 inhibits angiotensin II-induced vascular smooth muscle cell proliferation," Journal of the Renin-Angiotensin-Aldosterone System, vol. 15, no. 2, pp. 109-116, 2014.

[115] H. Duan, Y. Li, L. Yan et al., "MicroRNA-217 suppresses homocysteine-induced proliferation and migration of vascular smooth muscle cells via N-methyl-D-aspartic acid receptor inhibition," Clinical and Experimental Pharmacology and Physiology, vol. 43, no. 10, pp. 967-975, 2016.
[116] N. Choe, J.-S. Kwon, J.-R. Kim et al., “The microRNA miR-132 targets Lrrfip1 to block vascular smooth muscle cell proliferation and neointimal hyperplasia," Atherosclerosis, vol. 229, no. 2, pp. 348-355, 2013.

[117] X. Li, N. Yao, J. Zhang, and Z. Liu, "MicroRNA-125b is involved in atherosclerosis obliterans in vitro by targeting podocalyxin," Molecular Medicine Reports, vol. 12, no. 1, pp. 561-568, 2015.

[118] K. Kim, D. K. Yang, S. Kim, and H. Kang, "miR-142-3p is a regulator of the TGF $\beta$-mediated vascular smooth muscle cell phenotype," Journal of Cellular Biochemistry, vol. 116, no. 10, pp. 2325-2333, 2015.

[119] Y. Li, J. Huang, Z. Jiang et al., "MicroRNA-145 regulates plateletderived growth factor-induced human aortic vascular smooth muscle cell proliferation and migration by targeting CD40," American Journal of Translational Research, vol. 8, no. 4, pp. 1813-1825, 2016.

[120] B. Xie, C. Zhang, K. Kang, and S. Jiang, "miR-599 inhibits vascular smooth muscle cells proliferation and migration by targeting TGFB2," PLoS ONE, vol. 10, no. 11, Article ID e0141512, 2015.

[121] L. Qi, J. Zhi, T. Zhang et al., "Inhibition of microRNA-25 by tumor necrosis factor $\alpha$ is critical in the modulation of vascular smooth muscle cell proliferation," Molecular Medicine Reports, vol. 11, no. 6, pp. 4353-4358, 2015.

[122] C. Iaconetti, S. De Rosa, A. Polimeni et al., "Down-regulation of miR-23b induces phenotypic switching of vascular smooth muscle cells in vitro and in vivo," Cardiovascular Research, vol. 107, no. 4, pp. 522-533, 2015.

[123] F. Xu, A. S. I. Ahmed, X. Kang et al., "MicroRNA-15b/16 attenuates vascular neointima formation by promoting the contractile phenotype of vascular smooth muscle through targeting YAP," Arteriosclerosis, Thrombosis, and Vascular Biology, vol. 35, no. 10, pp. 2145-2152, 2015.

[124] J. Lee, S. Lim, B.-W. Song et al., "MicroRNA-29b inhibits migration and proliferation of vascular smooth muscle cells in neointimal formation," Journal of Cellular Biochemistry, vol. 116, no. 4, pp. 598-608, 2015.

[125] A. N. Qasim, S. S. Martin, N. N. Mehta et al., "Lipoprotein(a) is strongly associated with coronary artery calcification in type-2 diabetic women," International Journal of Cardiology, vol. 150, no. 1, pp. 17-21, 2011.

[126] W. G. Goodman, J. Goldin, B. D. Kuizon et al., "Coronary-artery calcification in young adults with end-stage renal disease who are undergoing dialysis," New England Journal of Medicine, vol. 342, no. 20, pp. 1478-1483, 2000.

[127] J.-K. Zhan, Y. Wang, J.-Y. He et al., "Artery calcification, osteoporosis, and plasma adiponectin levels in Chinese elderly," Heart and Lung, vol. 44, no. 6, pp. 539-543, 2015.

[128] J.-K. Zhan, Y.-J. Wang, Y. Wang et al., "The mammalian target of rapamycin signalling pathway is involved in osteoblastic differentiation of vascular smooth muscle cells," Canadian Journal of Cardiology, vol. 30, no. 5, pp. 568-575, 2014.

[129] L.-Q. Yuan, J.-H. Zhu, H.-W. Wang et al., "RANKL is a downstream mediator for insulin-induced osteoblastic differentiation of vascular smooth muscle cells," PLoS ONE, vol. 6, no. 12, Article ID e29037, 2011.

[130] P.-F. Shan, Y. Lu, R.-R. Cui, Y. Jiang, L.-Q. Yuan, and E.-Y. Liao, "Apelin attenuates the osteoblastic differentiation of vascular smooth muscle cells," PLoS ONE, vol. 6, no. 3, Article ID e17938, 2011.

[131] Q.-H. Liang, Y. Jiang, X. Zhu et al., "Ghrelin attenuates the osteoblastic differentiation of vascular smooth muscle cells 
through the ERK pathway," PLOS ONE, vol. 7, no. 4, Article ID e33126, 2012.

[132] J.-K. Zhan, Y.-J. Wang, Y. Wang et al., "Adiponectin attenuates the osteoblastic differentiation of vascular smooth muscle cells through the AMPK/mTOR pathway," Experimental Cell Research, vol. 323, no. 2, pp. 352-358, 2014.

[133] X.-B. Liao, X.-M. Zhou, J.-M. Li et al., "Taurine inhibits osteoblastic differentiation of vascular smooth muscle cells via the ERK pathway," Amino Acids, vol. 34, no. 4, pp. 525-530, 2008.

[134] G.-Y. Liu, Q.-H. Liang, R.-R. Cui et al., "Leptin promotes the osteoblastic differentiation of vascular smooth muscle cells from female mice by increasing RANKL expression," Endocrinology, vol. 155, no. 2, pp. 558-567, 2014.

[135] N. C. W. Mackenzie, K. A. Staines, D. Zhu, P. Genever, and V. E. Macrae, "miRNA-221 and miRNA-222 synergistically function to promote vascular calcification," Cell Biochemistry and Function, vol. 32, no. 2, pp. 209-216, 2014.

[136] R. Sudo, F. Sato, T. Azechi, and H. Wachi, "MiR-29-mediated elastin down-regulation contributes to inorganic phosphorusinduced osteoblastic differentiation in vascular smooth muscle cells," Genes to Cells, vol. 20, no. 12, pp. 1077-1087, 2015.

[137] L. Lin, Y. He, B.-L. Xi et al., "MiR-135a suppresses calcification in senescent VSMCs by regulating KLF4/STAT3 pathway," Current Vascular Pharmacology, vol. 14, no. 2, pp. 211-218, 2016.

[138] P. Wen, H. Cao, L. Fang et al., "MiR-125b/Ets1 axis regulates transdifferentiation and calcification of vascular smooth muscle cells in a high-phosphate environment," Experimental Cell Research, vol. 322, no. 2, pp. 302-312, 2014.

[139] W. Qiao, L. Chen, and M. Zhang, "MicroRNA-205 regulates the calcification and osteoblastic differentiation of vascular smooth muscle cells," Cellular Physiology and Biochemistry, vol. 33, no. 6, pp. 1945-1953, 2014.

[140] Z.-Y. Xia, Y. Hoo, P.-L. Xie et al., "Runx2/miR-3960/miR-2861 positive feedback loop is responsible for osteogenic transdifferentiation of vascular smooth muscle cells," BioMed Research International, vol. 2015, Article ID 624037, 7 pages, 2015.

[141] J. Wang, C.-H. Yan, Y. Li et al., "MicroRNA-31 controls phenotypic modulation of human vascular smooth muscle cells by regulating its target gene cellular repressor of E1A-stimulated genes," Experimental Cell Research, vol. 319, no. 8, pp. 1165-1175, 2013.

[142] S. Kim, A. Hata, and H. Kang, "Down-regulation of mir-96 by bone morphogenetic protein signaling is critical for vascular smooth muscle cell phenotype modulation," Journal of Cellular Biochemistry, vol. 115, no. 5, pp. 889-895, 2014.

[143] H. J. Kee, G. R. Kim, S.-N. Cho et al., "MiR-18a-5p microRNA increases vascular smooth muscle cell differentiation by downregulating syndecan4," Korean Circulation Journal, vol. 44, no. 4, pp. 255-263, 2014.

[144] P. Li, N. Zhu, B. Yi et al., "MicroRNA-663 regulates human vascular smooth muscle cell phenotypic switch and vascular neointimal formation," Circulation Research, vol. 113, no. 10, pp. 1117-1127, 2013.

[145] N. Zhao, S. N. Koenig, A. J. Trask et al., "MicroRNA miR145 regulates TGFBR2 expression and matrix synthesis in vascular smooth muscle cells," Circulation Research, vol. 116, no. 1, pp. 23-34, 2015.

[146] K. R. Cordes, N. T. Sheehy, M. P. White et al., "MiR-145 and miR143 regulate smooth muscle cell fate and plasticity," Nature, vol. 460, no. 7256, pp. 705-710, 2009.
[147] Y. Cheng, X. Liu, J. Yang et al., "MicroRNA-145, a novel smooth muscle cell phenotypic marker and modulator, controls vascular neointimal lesion formation," Circulation Research, vol. 105, no. 2, pp. 158-166, 2009.

[148] T. Gui, G. Zhou, Y. Sun et al., "MicroRNAs that target $\mathrm{Ca}^{2+}$ transporters are involved in vascular smooth muscle cell calcification," Laboratory Investigation, vol. 92, no. 9, pp. 1250-1259, 2012.

[149] J. A. F. Balderman, H.-Y. Lee, C. E. Mahoney et al., "Bone morphogenetic protein-2 decreases microRNA-30b and microRNA-30c to promote vascular smooth muscle cell calcification," Journal of the American Heart Association, vol. 1, no. 6, Article ID e003905, 2012.

[150] X. Zheng, A. Li, L. Zhao et al., "Key role of microRNA-15a in the KLF4 suppressions of proliferation and angiogenesis in endothelial and vascular smooth muscle cells," Biochemical and Biophysical Research Communications, vol. 437, no. 4, pp. 625631, 2013.

[151] S. Gilad, E. Meiri, Y. Yogev et al., "Serum microRNAs are promising novel biomarkers," PLoS ONE, vol. 3, no. 9, Article ID e3148, 2008.

[152] A. Zampetaki, P. Willeit, I. Drozdov, S. Kiechl, and M. Mayr, "Profiling of circulating microRNAs: from single biomarkers to re-wired networks," Cardiovascular Research, vol. 93, no. 4, pp. 555-562, 2012.

[153] T. Li, H. Cao, J. Zhuang et al., "Identification of miR-130a, miR-27b and miR-210 as serum biomarkers for atherosclerosis obliterans," Clinica Chimica Acta, vol. 412, no. 1-2, pp. 66-70, 2011.

[154] C. J. Creighton, J. G. Reid, and P. H. Gunaratne, "Expression profiling of microRNAs by deep sequencing," Briefings in Bioinformatics, vol. 10, no. 5, pp. 490-497, 2009.

[155] A. Git, H. Dvinge, M. Salmon-Divon et al., "Systematic comparison of microarray profiling, real-time PCR, and nextgeneration sequencing technologies for measuring differential microRNA expression," RNA, vol. 16, no. 5, pp. 991-1006, 2010.

[156] V. Köberle, T. Pleli, C. Schmithals et al., "Differential stability of cell-free circulating microRNAs: implications for their utilization as biomarkers," PLoS ONE, vol. 8, no. 9, Article ID e75184, 2013.

[157] P. Willeit, A. Zampetaki, K. Dudek et al., "Circulating microRNAs as novel biomarkers for platelet activation," Circulation Research, vol. 112, no. 4, pp. 595-600, 2013.

[158] J. M. Franco-Zorrilla, A. Valli, M. Todesco et al., "Target mimicry provides a new mechanism for regulation of microRNA activity", Nature Genetics, vol. 39, no. 8, pp. 1033-1037, 2007.

[159] J. Krützfeldt, N. Rajewsky, R. Braich et al., "Silencing of microRNAs in vivo with 'antagomirs"' Nature, vol. 438, no. 7068, pp. 685-689, 2005.

[160] K. Fluiter, O. R. Mook, and F. Baas, "The therapeutic potential of LNA-modified siRNAs: reduction of off-target effects by chemical modification of the siRNA sequence," in siRNA and miRNA Gene Silencing: From Bench to Bedside, vol. 487 of Methods in Molecular Biology, pp. 1-15, Springer, Berlin, Germany, 2009. 


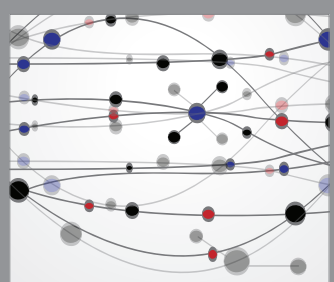

The Scientific World Journal
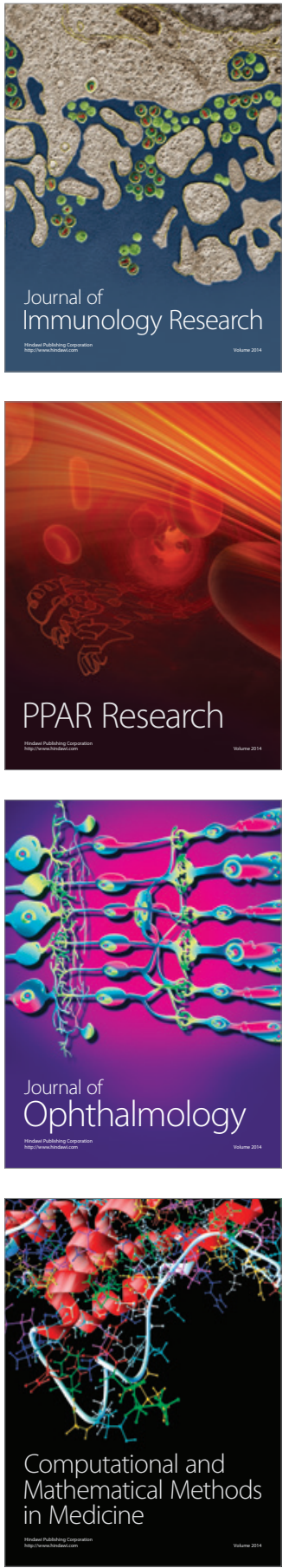

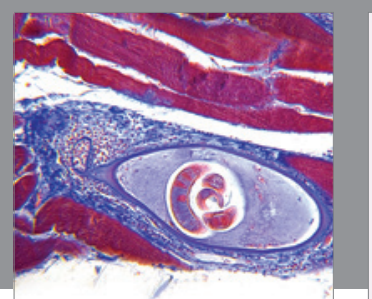

Gastroenterology Research and Practice

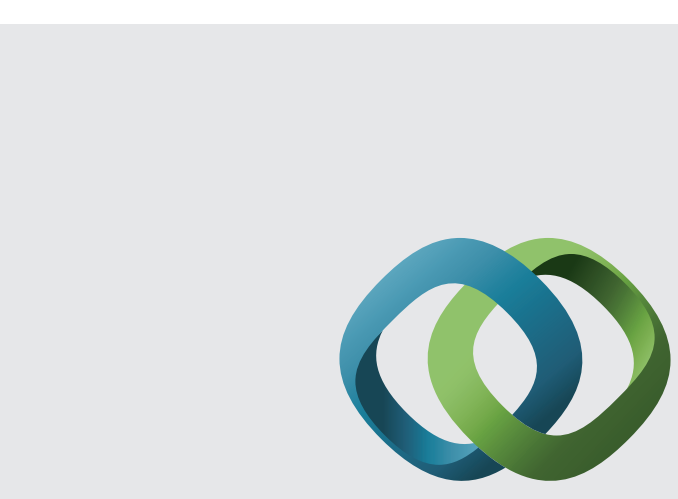

\section{Hindawi}

Submit your manuscripts at

http://www.hindawi.com
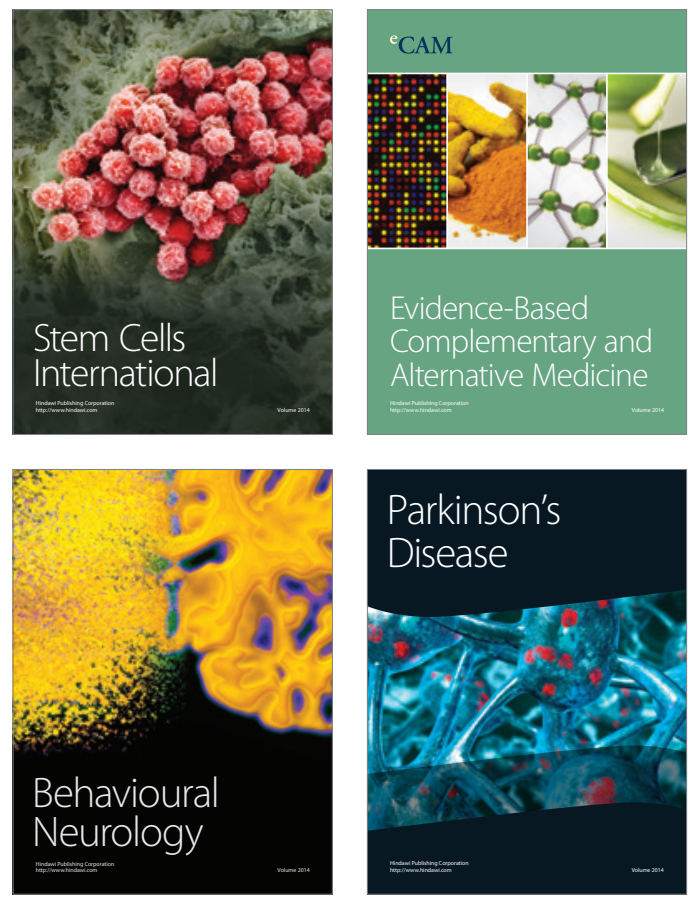
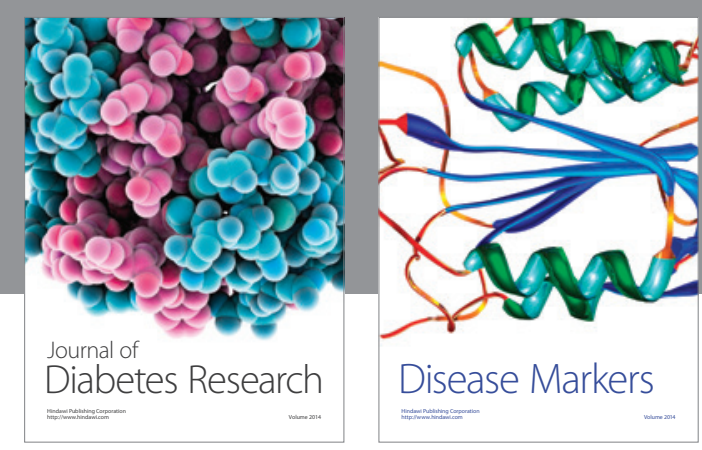

Disease Markers
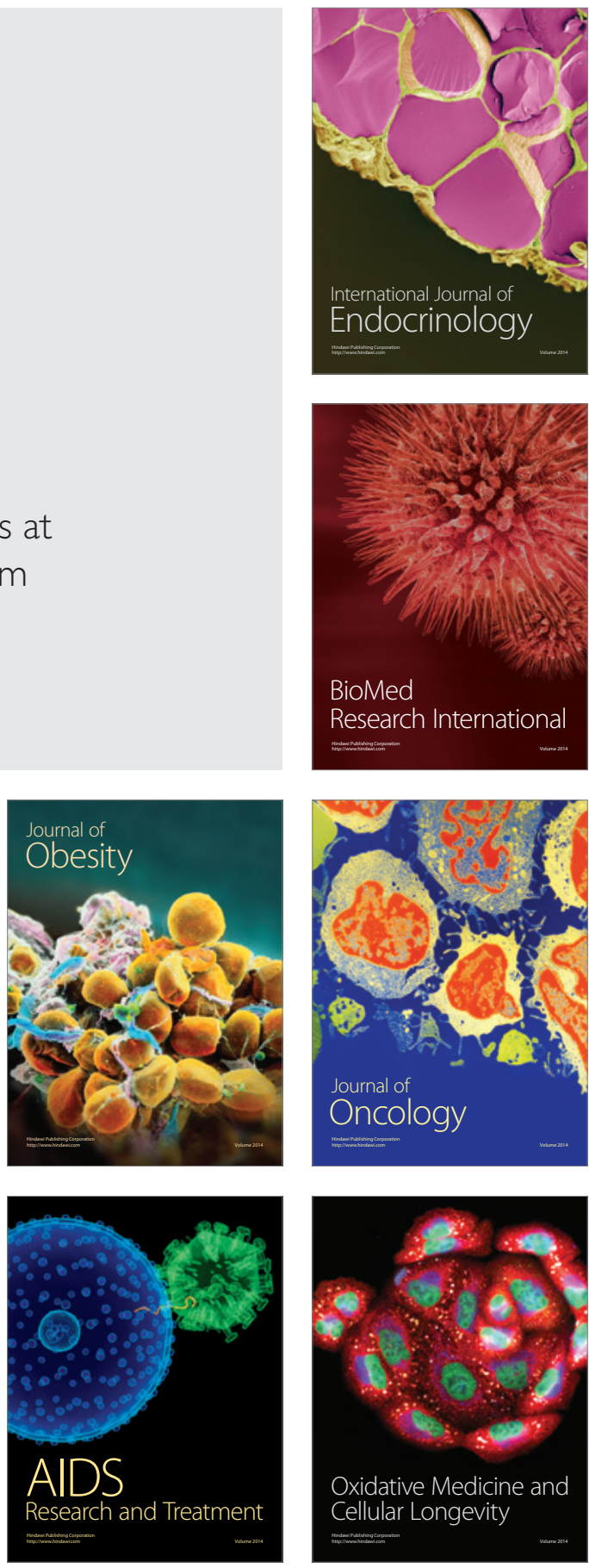